\title{
THE COMPLICATED DYNAMICS OF HEAVY RIGID BODIES ATTACHED TO DEFORMABLE RODS
}

\author{
By \\ STUART S. ANTMAN (Department of Mathematics and Institute for Physical Science and \\ Technology, University of Maryland, College Park, Maryland),
}

RANDALL S. MARLOW (2039 George Washington Way, Richland, Washington),

AND

CONSTANTINE P. VlahaCOS (Department of Physics, University of Maryland, College Park, Maryland)

\begin{abstract}
We study the motion in space of light nonlinearly elastic and viscoelastic rods with heavy rigid attachments. The rods, which can suffer flexure, extension, torsion, and shear, are described by a general geometrically exact theory. We pay particular attention to the leading term of the asymptotic expansion of the governing equations as the inertia of the rod goes to zero. When the rods are elastic and weightless, and when they have appropriate initial conditions, they move irregularly through a family of equilibrium states parametrized by time; the motion of the rigid body is governed by an interesting family of multivalued ordinary differential equations. These ordinary differential equations for a heavy mass point attached to an elastica undergoing planar motion are explicitly treated. These problems illuminate such phenomena as snap-buckling. On the other hand, when the rods are viscoelastic and weightless, the rigid body is typically not governed by ordinary differential equations, but, as we show, the motion of the system is well-defined for arbitrary initial conditions. This analysis relies critically on the careful use of our properly invariant constitutive hypotheses.
\end{abstract}

1. Introduction. In this paper we study the motion in space of light nonlinearly elastic and viscoelastic rods with heavy rigid attachments. We begin in Sec. 2 by formulating the geometrically exact equations for nonlinearly elastic and viscoelastic rods that can suffer flexure, extension, torsion, and shear. We then specialize our equations to rods in which the inertia of the rod is negligible relative to the inertia of the rigid attachment. We limit our attention here to the reduced problem (which is the leading term of the formal asymptotic expansion in a small parameter measuring the relative inertia) obtained by taking the inertias of the rod to be zero. 
In Sec. 3 we motivate our subsequent analysis by treating the very illuminating example of the planar motion of an elastica carrying a heavy mass at its end. The motion described by the reduced problem is one in which all the potential energy is possessed by the rod and all the kinetic energy is possessed by the end mass. The rod moves through a family of equilibrium states parametrized by time; these states can be explicitly characterized in terms of elliptic functions. The end mass is governed by a multivalued dynamics. Our detailed treatment of this problem suggests a very intriguing explanation and description of snap-buckling.

In Sec. 4 we treat the general problem for elastic rods, describing how the dynamics of the whole system is driven by the novel dynamics of the rigid attachment. Since the small inertia parameter multiplies time derivatives, one would expect that a full asymptotic analysis would indicate the presence of an initial layer. To have any chance of justifying such asymptotics, it seems necessary to include a suitable dissipative term. We introduce a general dissipative term in Sec. 5 , where we study nonlinearly viscoelastic rods. Their treatment differs considerably from that for elastic rods. We pay particular attention to the global solvability of the reduced equations for viscoelastic rods. This question is novel, but elementary, from the viewpoint of analysis. It is, however, complicated and illuminating from the viewpoint of mechanics: Its treatment requires the careful marshalling of constitutive hypotheses.

Our work represents the insertion of several pieces into a fascinating jigsaw puzzle corresponding to the full solution of a class of asymptotic problems that exhibit striking physical effects. Some of our results seem particularly applicable to space structures.

Notation. We employ Gibbs notation for vectors and tensors: Vectors, which are elements of Euclidean 3 -space $\mathbb{E}^{3}$, and vector-valued functions are denoted by lowercase, italic, bold-face symbols $\boldsymbol{u}, \boldsymbol{v}, \ldots$ The three vectors $\left\{\boldsymbol{i}_{1}, \boldsymbol{i}_{2}, \boldsymbol{i}_{3}\right\} \equiv\{\boldsymbol{i}, \boldsymbol{j}, \boldsymbol{k}\}$ are assumed to form a fixed right-handed orthonormal basis. The dot product of (vectors) $\boldsymbol{u}$ and $\boldsymbol{v}$ is denoted by $\boldsymbol{u} \cdot \boldsymbol{v}$. Tensors are linear transformations of $\mathbb{E}^{3}$ into itself. They are denoted by upper-case, italic, bold-face symbols $\boldsymbol{A}, \boldsymbol{B}, \ldots$ The value of tensor $\boldsymbol{A}$ at vector $\boldsymbol{v}$ is denoted $\boldsymbol{A} \cdot \boldsymbol{v}$ (in place of the more usual $\boldsymbol{A} \boldsymbol{v}$ ) and the product of $\boldsymbol{A}$ and $\boldsymbol{B}$ is denoted $\boldsymbol{A} \cdot \boldsymbol{B}$ (in place of the more usual $\boldsymbol{A} \boldsymbol{B}$ ). The transpose of $\boldsymbol{A}$ is denoted $\boldsymbol{A}^{*}$. We write $\boldsymbol{u} \cdot \boldsymbol{A}=\boldsymbol{A}^{*} \cdot \boldsymbol{u}$. The dyadic product of vectors $\boldsymbol{a}$ and $\boldsymbol{b}$ is denoted $\boldsymbol{a} \boldsymbol{b}$ (in place of the more usual $\boldsymbol{a} \otimes \boldsymbol{b})$. It is the tensor defined by $(\boldsymbol{a b}) \cdot \boldsymbol{u}=(\boldsymbol{b} \cdot \boldsymbol{u}) \boldsymbol{a}$ for all $\boldsymbol{u}$.

Triples of components of vectors are denoted by lower-case, bold-face roman symbols $\mathbf{u}, \mathbf{v}, \ldots$. Matrices of tensors are denoted by upper-case, bold-face roman symbols $\mathbf{A}, \mathbf{B}, \ldots$ We use the same notational scheme for operations involving these entities.

Twice-repeated lower-case Latin indices are summed from 1 to 3 and twice-repeated lower-case Green indices are summed from 1 to 2 .

The (Gâteaux) differential of $\boldsymbol{u} \mapsto \boldsymbol{f}(\boldsymbol{u})$ at $\boldsymbol{v}$ in the direction $\boldsymbol{h}$ is $\left.\frac{d}{d t} \boldsymbol{f}(\boldsymbol{v}+t \boldsymbol{h})\right|_{t=0}$. When it is linear in $\boldsymbol{h}$, we denote this differential by $\frac{\partial \boldsymbol{f}}{\partial \boldsymbol{u}}(\boldsymbol{v}) \cdot \boldsymbol{h}$ or $\boldsymbol{f}_{\boldsymbol{u}}(\boldsymbol{v}) \cdot \boldsymbol{h}$. We often denote the function $\boldsymbol{u} \mapsto \boldsymbol{f}(\boldsymbol{u})$ by $\boldsymbol{f}(\cdot)$. The partial derivative of a function $f$ with respect to a scalar argument $t$ is denoted by either $f_{t}$ or $\partial_{t} f$. Obvious analogs of these notations will also be used. In particular, $\partial \mathbf{f} / \partial \mathbf{u}$ is the matrix of partial derivatives of the components of $\mathbf{f}$ with respect to the components of $\mathbf{u}$. If $\mathbf{f}$ and $\mathbf{g}$ are three-component 
functions of the triples $\mathbf{u}$ and $\mathbf{v}$, then

$$
\frac{\partial(\mathbf{f}, \mathbf{g})}{\partial(\mathbf{u}, \mathbf{v})} \equiv\left(\begin{array}{ll}
\partial \mathbf{f} / \partial \mathbf{u} & \partial \mathbf{f} / \partial \mathbf{v} \\
\partial \mathbf{g} / \partial \mathbf{u} & \partial \mathbf{g} / \partial \mathbf{v}
\end{array}\right)
$$

is the $6 \times 6$ matrix consisting of the four indicated $3 \times 3$ submatrices.

2. Formulation of the governing equations. We give a brief formulation of the geometrically exact equations of motion of a rod that can suffer flexure, extension, torsion, and shear. (For this purpose we use a Cosserat theory of rods with an orthonormal triad of directors.) For full details and motivations, see [2].

Geometry of deformation. The motion of a rod is defined by three vector-valued functions

$$
[0,1] \times \mathbb{R} \ni(s, t) \mapsto \boldsymbol{r}(s, t), \boldsymbol{d}_{1}(s, t), \boldsymbol{d}_{2}(s, t) \in \mathbb{E}^{3}
$$

with $\boldsymbol{d}_{1}(s, t)$ and $\boldsymbol{d}_{2}(s, t)$ orthonormal. $\boldsymbol{r}(\cdot, t)$ may be interpreted as the configuration at time $t$ of any material curve connecting the "ends" of a slender three-dimensional body, e.g., a curve of centroids of the cross sections or a curve of mass centers of the cross sections of the body. $\boldsymbol{d}_{1}(s, t)$ and $\boldsymbol{d}_{2}(s, t)$ may be interpreted as characterizing the configuration of the material section (at) $s$ at time $t$. In particular, $\boldsymbol{d}_{1}(s, t)$ and $\boldsymbol{d}_{2}(s, t)$ may be regarded as characterizing the configurations of a pair of orthogonal material lines of the section $s$. We assume that $s$ is the arc-length parameter of the reference configuration $\stackrel{\circ}{r}$ of $\boldsymbol{r}$ and we scale the length so that $0 \leq s \leq 1$. We set

$$
\boldsymbol{d}_{3} \equiv \boldsymbol{d}_{1} \times \boldsymbol{d}_{2}
$$

Thus $\left\{\boldsymbol{d}_{k}(s, t)\right\}$ for each $(s, t)$ is a right-handed orthonormal basis for $\mathbb{E}^{3}$. These vectors are called directors. Their orthonormality implies that there are vector-valued functions $\boldsymbol{u}$ and $\boldsymbol{w}$ such that

$$
\partial_{s} \boldsymbol{d}_{k}=\boldsymbol{u} \times \boldsymbol{d}_{k}, \quad \partial_{t} \boldsymbol{d}_{k}=\boldsymbol{w} \times \boldsymbol{d}_{k} .
$$

Since the basis $\left\{\boldsymbol{d}_{k}\right\}$ is natural for the intrinsic description of deformation, we decompose relevant vector-valued functions with respect to it:

$$
\boldsymbol{u}=u_{k} \boldsymbol{d}_{k}, \quad \boldsymbol{r}_{s}=v_{k} \boldsymbol{d}_{k}, \quad \boldsymbol{w}=w_{k} \boldsymbol{d}_{k} .
$$

By using the equality of mixed partial derivatives of the $\boldsymbol{d}_{k}$, we can prove that

$$
\boldsymbol{w}_{s}=\boldsymbol{u}_{t}+\boldsymbol{u} \times \boldsymbol{w}=\left(\partial_{t} u_{k}\right) \boldsymbol{d}_{k} .
$$

The triples

$$
\mathbf{u} \equiv\left(u_{1}, u_{2}, u_{3}\right), \quad \mathbf{v} \equiv\left(v_{1}, v_{2}, v_{3}\right)
$$

are the strain variables corresponding to the motion (2.1). For fixed $t$ the functions $\mathbf{u}(\cdot, t)$ and $\mathbf{v}(\cdot, t)$ determine $\boldsymbol{r}(\cdot, t), \boldsymbol{d}_{1}(\cdot, t), \boldsymbol{d}_{2}(\cdot, t)$ (the configuration at time $t$ ) to within a rigid motion and thus account for the change of shape. The strains $u_{1}$ and $u_{2}$ measure flexure, $u_{3}$ measures torsion, $v_{1}$ and $v_{2}$ measure shear, and $v_{3}$ measures dilatation.

Under our interpretations of the kinematic variables, a rod-theoretic analog of the three-dimensional requirement that the Jacobian of the deformation be positive (so that orientation is preserved) is that there be a function $\left(u_{1}, u_{2}, s\right) \mapsto V\left(u_{1}, u_{2}, s\right)$ for which 
$V(0,0, s)=0, V\left(u_{1}, u_{2}, s\right)>0$ for $u_{\alpha} u_{\alpha}>0$, and $V(\cdot, \cdot, s)$ is convex and homogeneous of degree 1 such that

$$
v_{3}>V\left(u_{1}, u_{2}, s\right)
$$

Thus for each $s$ the surface $v_{3}=V\left(u_{1}, u_{2}, s\right)$ is a cone in $\left(u_{1}, u_{2}, v_{3}\right)$-space. (E.g., for a rod with a circular cross section at $s$ of radius $h(s)$ at $s$ the function $V$ reduces to $V\left(u_{1}, u_{2}, s\right)=h(s) \sqrt{\left(u_{1}\right)^{2}+\left(u_{2}\right)^{2}}$.) A consequence of $(2.7)$ is that

$$
v_{3}=\boldsymbol{r}_{s} \cdot \boldsymbol{d}_{3}>0 \text {. }
$$

This condition implies that (i) $\left|\boldsymbol{r}_{s}\right|>0$, so that the local ratio of deformed to reference length of the axis cannot be reduced to zero, and (ii) a typical section $s$ cannot undergo a total shear in which the plane determined by $\boldsymbol{d}_{1}(s, t)$ and $\boldsymbol{d}_{2}(s, t)$ is tangent to the curve $\boldsymbol{r}(\cdot, t)$ at $\boldsymbol{r}(s, t)$.

We identify geometric quantities in the reference configuration by a superposed circle. We assume that $\stackrel{\circ}{v}_{1}=0=\stackrel{\circ}{v}_{2}, \stackrel{\circ}{v}_{3}=1$.

We assume that the end $s=0$ of the rod is welded to the $\{\boldsymbol{i}, \boldsymbol{j}\}$-plane, so that

$$
\boldsymbol{r}(0, t)=\mathbf{0}, \quad \boldsymbol{d}_{k}(0, t)=\boldsymbol{i}_{k} .
$$

We restrict our attention to motions for which there is no self-contact.

Mechanics. In the configuration at time $t$, the resultant contact force and contact couple exerted by the material of $[0, s)$ on the material of $[s, 1]$ (for $0<s \leq 1$ ) are respectively denoted $\boldsymbol{- n}(s, t)$ and $\boldsymbol{-} \boldsymbol{m}(s, t)$. At $(s, t)$ the rod is subjected to a body force of intensity $\boldsymbol{f}(s, t)$ and a body couple of intensity $\boldsymbol{l}(s, t)$ per unit reference length at $(s, t)$.

Motivated in part by three-dimensional considerations in which $\boldsymbol{r}$ is the material curve of centroids of the three-dimensional rod we assume that the linear and angular momenta per unit reference length at $(s, t)$ have the forms $\varepsilon(\rho A)(s) \boldsymbol{r}_{t}(s, t)$ and $\chi(\varepsilon, s) \boldsymbol{h}(s, t)$ where

$$
\boldsymbol{h}(s, t) \equiv(\rho \boldsymbol{I})(s, t) \cdot \boldsymbol{w}(s, t) \equiv\left(\rho I_{p q}\right)(s) w_{q}(s, t) \boldsymbol{d}_{p}(s, t) .
$$

We take $\varepsilon$ to be a nonnegative parameter and $\chi$ to be a prescribed function with $\chi(\varepsilon, s)>$ 0 for $\varepsilon>0$ and $0<s<1$, and with $\chi(0, s)=0$. ( $\chi$ is determined by the cross-sectional shape.) The parameter $\varepsilon$ is a measure of the size of the inertias of the rod relative to the size of the inertias of the rigid body. It is not a thickness parameter. The function $\rho A$ is prescribed to be positive-valued. It may be interpreted as the scaled mass density per reference length. The $\left(\rho I_{\gamma \delta}\right)(s)$ are the prescribed components of a positive-definite symmetric $2 \times 2$ matrix, which may be thought of as a matrix of scaled mass-moments of inertia of the section $s, \rho I_{\gamma 3}=\rho I_{3 \gamma}=0, \rho I_{33}=\rho I_{\gamma \gamma}$, and $\rho \boldsymbol{I} \equiv I_{p q} \boldsymbol{d}_{p} \boldsymbol{d}_{q}$. Inherent in the form of (2.10) is an assumption that $\boldsymbol{r}$ is chosen appropriately so that there are no other contributions to (2.10) involving first mass-moments of the cross section. For a straight, uniform, prismatic rod, (2.10) is valid when $\boldsymbol{r}$ is the curve of centroids. The methods for treating more general forms of (2.10) are identical to those used here.

We assume that a rigid body of mass $\mu$ is rigidly attached to the end $s=1$ of the rod. Its mass center is at $\boldsymbol{r}(1, t)+\boldsymbol{c}(t)$, where, because of the rigidity of the attachment, $\boldsymbol{c}(t)$ has the form $c_{k} \boldsymbol{d}_{k}(1, t)$, with the $c_{k}$ prescribed constants. This rigid body has inertia tensor $\boldsymbol{J}(t)=J_{k l} \boldsymbol{d}_{k}(1, t) \boldsymbol{d}_{l}(1, t)$ about its mass center. Here the $J_{k l}$ are the constant 
components of a positive-definite matrix. For simplicity of exposition, we assume that there are no external forces or couples applied to the rigid body by any agent other than the rod.

For the body consisting of the segment $[s, 1]$ of the rod and the rigid body, the requirement that the impulse of forces over the time interval $[0, \tau]$ acting on the body equal the change in linear momentum of this body over this time interval yields

$$
\begin{aligned}
-\int_{0}^{\tau} \boldsymbol{n}(s, t) d t+ & \int_{0}^{\tau} \int_{s}^{1} \boldsymbol{f}(\xi, t) d \xi d t \\
& =\left.\int_{s}^{1} \varepsilon(\rho A)(\xi) \boldsymbol{r}_{t}(\xi, t) d \xi\right|_{t=0} ^{t=\tau}+\left.\mu\left[\boldsymbol{r}_{t}(1, t)+\boldsymbol{c}_{t}(t)\right]\right|_{t=0} ^{t=\tau}
\end{aligned}
$$

for all $s$ and $\tau$. Similarly, by requiring that the impulse of torques about $\mathbf{0}$ equal the change in angular momentum about $\mathbf{0}$, we obtain

$$
\begin{aligned}
& -\int_{0}^{\tau}[\boldsymbol{m}(s, t)+\boldsymbol{r}(s, t) \times \boldsymbol{n}(s, t)] d t \\
& +\int_{0}^{\tau} \int_{s}^{1}[\boldsymbol{r}(\xi, t) \times \boldsymbol{f}(\xi, t)+\boldsymbol{l}(\xi, t)] d \xi d t \\
& \quad=\left.\int_{s}^{1}\left\{\varepsilon \boldsymbol{r}(\xi, t) \times(\rho A)(\xi) \boldsymbol{r}_{t}(\xi, t)+\chi(\varepsilon, s) \boldsymbol{h}(\xi, t)\right\} d \xi\right|_{t=0} ^{t=\tau} \\
& \quad+\left.\left\{\mu[\boldsymbol{r}(1, t)+\boldsymbol{c}(t)] \times\left[\boldsymbol{r}_{t}(1, t)+\boldsymbol{c}_{t}(t)\right]+\boldsymbol{J}(t) \cdot \boldsymbol{w}(1, t)\right\}\right|_{t=0} ^{t=\tau}
\end{aligned}
$$

for all $s$ and $\tau$.

Assuming that all the functions appearing in (2.11) and (2.12) are sufficiently regular, we can differentiate these equations with respect to $s$ and $\tau$ to obtain the classical forms of the equations of motion:

$$
\begin{gathered}
\boldsymbol{n}_{s}+\boldsymbol{f}=\varepsilon \rho A \boldsymbol{r}_{t t}, \\
\boldsymbol{m}_{s}+\boldsymbol{r}_{s} \times \boldsymbol{n}+\boldsymbol{l}=\chi(\varepsilon, s) \boldsymbol{h}_{t} .
\end{gathered}
$$

By letting $s \rightarrow 1$ in (2.11) and (2.12) and then differentiating these equations with respect to $\tau$, we obtain the important boundary conditions

$$
\begin{aligned}
-\boldsymbol{n}(1, t) & =\mu\left[\boldsymbol{r}_{t t}(1, t)+\boldsymbol{c}_{t t}(t)\right], \\
-\boldsymbol{m}(1, t)+\boldsymbol{c}(t) \times \boldsymbol{n}(1, t) & =[\boldsymbol{J}(t) \cdot \boldsymbol{w}(1, t)]_{t} .
\end{aligned}
$$

Note that

$$
\begin{gathered}
\boldsymbol{c}_{t}(t)=\boldsymbol{w}(1, t) \times \boldsymbol{c}(t), \quad \boldsymbol{w}_{t}(1, t)=\partial_{t} w_{k}(1, t) \boldsymbol{d}_{k}(1, t), \\
{[\boldsymbol{J}(t) \cdot \boldsymbol{w}(1, t)]_{t}=\boldsymbol{J}(t) \cdot \boldsymbol{w}_{t}(1, t)+\boldsymbol{w}(1, t) \times[\boldsymbol{J}(t) \cdot \boldsymbol{w}(1, t)]}
\end{gathered}
$$

Let

$$
\mathbf{m} \equiv\left(m_{1}, m_{2}, m_{3}\right), \mathbf{n} \equiv\left(n_{1}, n_{2}, n_{3}\right) \quad \text { where } m_{k} \equiv \boldsymbol{m} \cdot \boldsymbol{d}_{k}, n_{k} \equiv \boldsymbol{n} \cdot \boldsymbol{d}_{k} .
$$

We may call $m_{1}$ and $m_{2}$ the bending couples, $m_{3}$ the twisting couple, $n_{1}$ and $n_{2}$ the shear forces, and $\boldsymbol{n} \cdot \boldsymbol{r}_{\boldsymbol{s}} /\left|\boldsymbol{r}_{s}\right|$ the tension. These terms are not strictly analogous to those used in structural mechanics, in which it is usually assumed that $\boldsymbol{d}_{3}=\boldsymbol{r}_{s} /\left|\boldsymbol{r}_{s}\right|$. 
Constitutive equations. The rod is called elastic if there are constitutive functions $(\mathbf{u}, \mathbf{v}, s) \mapsto \hat{\mathbf{m}}(\mathbf{u}, \mathbf{v}, s)$ and $(\mathbf{u}, \mathbf{v}, s) \mapsto \hat{\mathbf{n}}(\mathbf{u}, \mathbf{v}, s)$ such that

$$
\mathbf{m}(s, t)=\hat{\mathbf{m}}(\mathbf{u}(s, t), \mathbf{v}(s, t), s), \quad \mathbf{n}(s, t)=\hat{\mathbf{n}}(\mathbf{u}(s, t), \mathbf{v}(s, t), s) .
$$

For each fixed $s$, the common domain of these constitutive functions is the subset of $\mathbf{u}$ 's and v's that satisfy (2.7). The rod is called viscoelastic of strain-rate type of complexity 1 if there are constitutive functions $(\mathbf{u}, \mathbf{v}, \dot{\mathbf{u}}, \dot{\mathbf{v}}, s) \mapsto \hat{\mathbf{m}}(\mathbf{u}, \mathbf{v}, \dot{\mathbf{u}}, \dot{\mathbf{v}}, s)$ and $(\mathbf{u}, \mathbf{v}, \dot{\mathbf{u}}, \dot{\mathbf{v}}, s) \mapsto$ $\hat{\mathbf{n}}(\mathbf{u}, \mathbf{v}, \dot{\mathbf{u}}, \dot{\mathbf{v}}, s)$ such that

$$
\begin{aligned}
\mathbf{m}(s, t) & =\hat{\mathbf{m}}\left(\mathbf{u}(s, t), \mathbf{v}(s, t), \mathbf{u}_{t}(s, t), \mathbf{v}_{t}(s, t), s\right), \\
\mathbf{n}(s, t) & =\hat{\mathbf{n}}\left(\mathbf{u}(s, t), \mathbf{v}(s, t), \mathbf{u}_{t}(s, t), \mathbf{v}_{t}(s, t), s\right) .
\end{aligned}
$$

The dots over $\mathbf{u}$ and $\mathbf{v}$ have mnemonic, but not operational, significance: They merely identify the third and fourth arguments of $\hat{\mathbf{m}}$ and $\hat{\mathbf{n}}$ in (2.19). The common domain of these constitutive functions is also defined by (2.7). These forms of the constitutive equations ensure that the material response is unaffected by rigid motions. For simplicity of exposition we assume that $\hat{\mathbf{m}}$ and $\hat{\mathbf{n}}$ are continuously differentiable.

Restrictions for elastic rods. If the constitutive equations (2.19) for elastic rods are to conform to our intuition and experience, they should meet some minimal physical restrictions. We accordingly require that an increase in the tension $\boldsymbol{n} \cdot \boldsymbol{r}_{s} /\left|\boldsymbol{r}_{s}\right|$ accompany an increase in the stretch $\left|\boldsymbol{r}_{s}\right|$, an increase in a shear component $n_{\alpha}$ of the contact force accompany an increase in the shear strain $v_{\alpha}$, an increase in a bending couple $m_{\alpha}$ accompany an increase in the flexure $u_{\alpha}$, and an increase in the twisting couple $m_{3}$ accompany an increase in the twist $u_{3}$. These conditions are ensured by the requirement:

$$
\frac{\partial(\hat{\mathbf{m}}, \hat{\mathbf{n}})}{\partial(\mathbf{u}, \mathbf{v})} \text { is positive-definite. }
$$

(This condition is equivalent to the positive-definiteness of the symmetric part of this matrix.) We adopt (2.21) even though much of our analysis could be extended to problems in which it is suspended (cf. [6, 9]).

We assume that the elastic rod is hyperelastic (i.e., that the matrix of $(2.21)$ is symmetric), so that there is a scalar-valued stored-energy function $W$ such that

$$
\hat{\mathbf{m}}(\mathbf{u}, \mathbf{v}, s)=\frac{\partial W}{\partial \mathbf{u}}(\mathbf{u}, \mathbf{v}, s), \quad \hat{\mathbf{n}}(\mathbf{u}, \mathbf{v}, s)=\frac{\partial W}{\partial \mathbf{v}}(\mathbf{u}, \mathbf{v}, s) .
$$

Condition (2.21) ensures that $W(\cdot, \cdot, s)$ is strictly convex. We assume that $W \geq 0$.

We require that

$$
\hat{\mathbf{m}} \cdot(\mathbf{u}-\stackrel{\circ}{\mathbf{u}})+\hat{\mathbf{n}} \cdot(\mathbf{v}-\stackrel{\circ}{\mathbf{v}}) \rightarrow \infty \quad \text { as }|\mathbf{u}|+|\mathbf{v}| \rightarrow \infty \quad \text { or as } v_{3}-V\left(u_{1}, u_{2}, s\right) \rightarrow 0 .
$$

More specifically, we always assume that $W$ is strictly superlinear:

$$
\frac{W(\mathbf{u}, \mathbf{v}, s)}{\sqrt{|\mathbf{u}|^{2}+|\mathbf{v}|^{2}}} \rightarrow \infty \quad \text { as }|\mathbf{u}|^{2}+|\mathbf{v}|^{2} \rightarrow \infty
$$

In this case, there are numbers $c, C>0$ such that

$$
W(\mathbf{u}, \mathbf{v}, s) \geq c(|\mathbf{u}|+|\mathbf{v}|)-C .
$$


We may further require that a total compression result in an infinite energy:

$$
W(\mathbf{u}, \mathbf{v}, s) \rightarrow \infty \quad \text { as } v_{3}-V\left(u_{1}, u_{2}, s\right) \rightarrow 0 .
$$

An alternative penalization of total compression in the context of viscoelasticity is given below.

The set of conditions (2.21), (2.23) and the set of conditions (2.21), (2.24), (2.26) each support global implicit-function theorems that assert that the system of finitedimensional equations

$$
\hat{\mathbf{m}}(\mathbf{u}, \mathbf{v}, s)=\mathbf{m}, \quad \hat{\mathbf{n}}(\mathbf{u}, \mathbf{v}, s)=\mathbf{n}
$$

can be solved uniquely for $\mathbf{u}$ and $\mathbf{v}$ in terms of the other variables:

$$
\mathbf{u}=\hat{\mathbf{u}}(\mathbf{m}, \mathbf{n}, s), \quad \mathbf{v}=\hat{\mathbf{v}}(\mathbf{m}, \mathbf{n}, s)
$$

with $\hat{\mathbf{u}}$ and $\hat{\mathbf{v}}$ inheriting the regularity of $\hat{\mathbf{m}}$ and $\hat{\mathbf{n}}$ and satisfying the monotonicity condition:

$$
\frac{\partial(\hat{\mathbf{u}}, \hat{\mathbf{v}})}{\partial(\mathbf{m}, \mathbf{n})} \text { is positive-definite. }
$$

The equivalence of (2.27) and (2.28) implies that (2.19) is equivalent to

$$
\mathbf{u}(s, t)=\hat{\mathbf{u}}(\mathbf{m}(s, t), \mathbf{n}(s, t), s), \quad \mathbf{v}(s, t)=\hat{\mathbf{v}}(\mathbf{m}(s, t), \mathbf{n}(s, t), s) .
$$

Moreover, we readily find that

$$
\hat{\mathbf{u}}=\frac{\partial W^{*}}{\partial \mathbf{m}}, \quad \hat{\mathbf{v}}=\frac{\partial W^{*}}{\partial \mathbf{n}}
$$

where $W^{*}$ is the convex function conjugate to $W$ :

$$
W^{*}(\mathbf{m}, \mathbf{n}, s) \equiv \mathbf{m} \cdot \hat{\mathbf{u}}(\mathbf{m}, \mathbf{n}, s)+\mathbf{n} \cdot \hat{\mathbf{v}}(\mathbf{m}, \mathbf{n}, s)-W(\hat{\mathbf{u}}(\mathbf{m}, \mathbf{n}, s), \hat{\mathbf{v}}(\mathbf{m}, \mathbf{n}, s), s) .
$$

The existence of $(2.28)$ follows from a standard proof that the function $(\mathbf{u}, \mathbf{v}) \mapsto$ $W(\mathbf{u}, \mathbf{v}, s)-\mathbf{u} \cdot \mathbf{m}+\mathbf{v} \cdot \mathbf{n}$ has a minimum on its domain defined by $(2.7)$, and that $(2.21)$ ensures that this minimum is unique. The classical Local Implicit-Function Theorem ensures that (2.28) inherits the regularity of (2.22).

Restrictions for viscoelastic rods. For viscoelastic rods, we require that $\hat{\mathbf{m}}(\cdot, \cdot, \mathbf{0}, \mathbf{0}, s)$ and $\hat{\mathbf{n}}(\cdot, \cdot, \mathbf{0}, \mathbf{0}, s)$ have exactly the same properties as those we have just imposed on the functions $\hat{\mathbf{m}}(\cdot, \cdot, s)$ and $\hat{\mathbf{n}}(\cdot, \cdot, s)$ for elastic rods, except for $(2.21)$, which implies the convexity of $W(\cdot, \cdot, s)$, because it is not necessary for our analysis. (This convexity is reasonable, but restrictive; it prohibits the circumstances that lead to much of the modern work on the continuum theory of phase changes.)

We further require that effects of internal friction grow with the strain rates:

$$
\frac{\partial(\hat{\mathbf{m}}, \hat{\mathbf{n}})}{\partial(\dot{\mathbf{u}}, \dot{\mathbf{v}})} \text { is positive-definite. }
$$

This monotonicity condition ensures that the response is truly dissipative and that the governing equations of motion have a parabolic character. We further assume that infinite 
strain rates produce infinite resultants:

$$
\frac{[\hat{\mathbf{m}}(\mathbf{u}, \mathbf{v}, \dot{\mathbf{u}}, \dot{\mathbf{v}}, s)-\hat{\mathbf{m}}(\mathbf{u}, \mathbf{v}, \mathbf{0}, \mathbf{0}, s)] \cdot \dot{\mathbf{u}}+[\hat{\mathbf{n}}(\mathbf{u}, \mathbf{v}, \dot{\mathbf{u}}, \dot{\mathbf{v}}, s)-\hat{\mathbf{n}}(\mathbf{u}, \mathbf{v}, \mathbf{0}, \mathbf{0}, s)] \cdot \dot{\mathbf{v}}}{\sqrt{|\dot{\mathbf{u}}|^{2}+|\dot{\mathbf{v}}|^{2}}} \rightarrow \infty
$$

for bounded $\mathbf{u}, \mathbf{v}$ satisfying (2.7). In this case, there are numbers $c, C>0$ such that

$$
\begin{array}{r}
{[\hat{\mathbf{m}}(\mathbf{u}, \mathbf{v}, \dot{\mathbf{u}}, \dot{\mathbf{v}}, s)-\hat{\mathbf{m}}(\mathbf{u}, \mathbf{v}, \mathbf{0}, \mathbf{0}, s)] \cdot \dot{\mathbf{u}}+[\hat{\mathbf{n}}(\mathbf{u}, \mathbf{v}, \dot{\mathbf{u}}, \dot{\mathbf{v}}, s)-\hat{\mathbf{n}}(\mathbf{u}, \mathbf{v}, \mathbf{0}, \mathbf{0}, s)] \cdot \mathbf{v}} \\
\geq c(|\dot{\mathbf{u}}|+|\dot{\mathbf{v}}|)-C .
\end{array}
$$

Conditions (2.33) and (2.34) support a global implicit-function theorem (based on degree theory) that asserts that the system of finite-dimensional equations

$$
\hat{\mathbf{m}}(\mathbf{u}, \mathbf{v}, \dot{\mathbf{u}}, \dot{\mathbf{v}}, s)=\mathbf{m}, \quad \hat{\mathbf{n}}(\mathbf{u}, \mathbf{v}, \dot{\mathbf{u}}, \dot{\mathbf{v}}, s)=\mathbf{n}
$$

can be solved uniquely for $\dot{\mathbf{u}}$ and $\dot{\mathbf{v}}$ in terms of the other variables:

$$
\dot{\mathbf{u}}=\dot{\mathbf{u}}^{\sharp}(\mathbf{u}, \mathbf{v}, \mathbf{m}, \mathbf{n}, s), \quad \dot{\mathbf{v}}=\dot{\mathbf{v}}^{\sharp}(\mathbf{u}, \mathbf{v}, \mathbf{m}, \mathbf{n}, s)
$$

with $\dot{\mathbf{u}}^{\sharp}$ and $\dot{\mathbf{v}}^{\sharp}$ inheriting the regularity of $\hat{\mathbf{m}}$ and $\hat{\mathbf{n}}$ and satisfying the monotonicity condition:

$$
\frac{\partial\left(\dot{\mathbf{u}}^{\sharp}, \dot{\mathbf{v}}^{\sharp}\right)}{\partial(\dot{\mathbf{m}}, \dot{\mathbf{n}})} \text { is positive-definite. }
$$

The equivalence of (2.36) and (2.37) implies that (2.20) is equivalent to

$$
\mathbf{u}_{t}(s, t)=\dot{\mathbf{u}}^{\sharp}(\mathbf{u}(s, t), \mathbf{v}(s, t), \mathbf{m}(s, t), \mathbf{n}(s, t), s), \ldots
$$

We can require that frictional effects become infinitely large in a suitable way at a total compression. (Such an assumption plays a crucial role in the global existence theory of [3] for a scalar problem.) To describe such a condition we define

$$
\delta^{\sharp}(\mathbf{u}, \mathbf{v}, s) \equiv v_{3}-V\left(u_{1}, u_{2}, s\right), \quad \dot{\delta}^{\sharp}(\mathbf{u}, \mathbf{v}, \dot{\mathbf{u}}, \dot{\mathbf{v}}, s) \equiv \dot{v}_{3}-\frac{\partial V}{\partial u_{\alpha}}\left(u_{1}, u_{2}, s\right) \dot{u}_{\alpha} .
$$

We assume that there is a positive number $\eta$, a constant $C>0$, and a continuously differentiable function $(0, \eta) \times[0,1] \ni(\delta, s) \mapsto \Omega(\delta, s)$ with $\Omega(\delta, s) \rightarrow \infty$ as $\delta \rightarrow 0$ such that

$$
\hat{n}_{3}(\mathbf{u}, \mathbf{v}, \dot{\mathbf{u}}, \dot{\mathbf{v}}, s) \leq-\Omega_{\delta}\left(\delta^{\sharp}(\mathbf{u}, \mathbf{v}, s), s\right) \dot{\delta}^{\sharp}(\mathbf{u}, \mathbf{v}, \dot{\mathbf{u}}, \dot{\mathbf{v}}, s)+C \text { if } 0<\delta^{\sharp}(\mathbf{u}, \mathbf{v}, s) \leq \eta .
$$

Condition (2.41) is restrictive for large values of $\dot{\delta}$. It can be weakened at the expense of a much more complicated analysis. (See [3,4].)

The initial-boundary-value problem. We impose initial conditions on $\boldsymbol{r}, \boldsymbol{r}_{t}, \boldsymbol{d}_{k}, \partial_{t} \boldsymbol{d}_{k}$ that we assume are compatible with the boundary conditions at $s=0$. Then our initialboundary-value problem for a viscoelastic rod consists of the kinematic relations (2.3)(2.5), the equations of motion (2.13), (2.14), the constitutive equations (2.19) or (2.20), the boundary conditions (2.9), (2.15), (2.16), and the initial conditions just described. We take $\boldsymbol{f}=\mathbf{0}=\boldsymbol{l}$ to simplify our presentation. 
The reduced initial-boundary-value problem. Our interest in this paper is in the reduced initial-boundary-value problem, obtained by setting $\varepsilon=0$. In this case, from (2.13) and (2.14) we obtain the integrals

$$
\boldsymbol{n}(s, t)=\boldsymbol{n}(1, t), \quad \boldsymbol{m}(s, t)+\boldsymbol{r}(s, t) \times \boldsymbol{n}(1, t)=\boldsymbol{m}(1, t)+\boldsymbol{r}(1, t) \times \boldsymbol{n}(1, t),
$$

which are very useful for our study.

3. Example. The reduced problem for the elastica. A main motivation for our analysis is given by the problem for the elastica, which we now derive. Let us drastically specialize the reduced problem for elastic rods by introducing a host of simplifying assumptions. We assume that the problem is planar by requiring that $\boldsymbol{r} \cdot \boldsymbol{j}=0$ and $\boldsymbol{d}_{2}=\boldsymbol{j}$. We may accordingly introduce an angle $\theta$ such that

$$
\boldsymbol{d}_{3}=\cos \theta \boldsymbol{k}+\sin \theta \boldsymbol{i}, \quad \boldsymbol{d}_{1}=-\sin \theta \boldsymbol{k}+\cos \theta \boldsymbol{i} .
$$

In this case we immediately find that $\mathbf{u}=\left(0, \theta_{s}, 0\right)$. We take the rod to be unshearable and inextensible, so that $\mathbf{v}=(0,0,1)$. (This restriction is consistent with constitutive equations of the form (2.28).) We assume that the rod has enough symmetry that $\boldsymbol{m}=m_{2} \boldsymbol{j}$ when the strains meet the restrictions just imposed. We assume that our constitutive equations give $m_{2}$ as a constant multiple of $u_{2}$. (This is the Bernoulli-Euler constitutive assumption.) Without loss of generality, we scale the problem so that this multiple is unity. Finally we assume that the rigid body has no rotatory inertia about the free end of the rod: $\boldsymbol{c}=\mathbf{0}, \boldsymbol{J}=\boldsymbol{O}$.

Let us set

$$
\boldsymbol{n}(1, t)=-\lambda(t)^{2}[\cos \alpha(t) \boldsymbol{k}+\sin \alpha(t) \boldsymbol{i}] .
$$

We take $-\pi \leq \alpha(t) \leq \pi$. We substitute (2.42a) and (3.2) into the reduced version of (3.14) to get

$$
\theta_{s s}(s, t)+\lambda(t)^{2} \sin (\theta(s, t)-\alpha(t))=0 .
$$

From $(2.9 \mathrm{~b})$ and $(2.16)$ we obtain

$$
\theta(0, t)=0, \quad \theta_{s}(1, t)=0 .
$$

Conditions (2.4c) and (2.9a) yield

$$
\boldsymbol{r}(s, t) \equiv x(s, t) \boldsymbol{k}+y(s, t) \boldsymbol{i}=\int_{0}^{s}[\cos \theta(\xi, t) \boldsymbol{k}+\sin \theta(\xi, t) \boldsymbol{i}] d \xi .
$$

The substitution of (3.2) into (2.15) yields

$$
\lambda(t)^{2}[\cos \alpha(t) \boldsymbol{k}+\sin \alpha(t) \boldsymbol{i}]=\mu \boldsymbol{r}_{t t}(1, t) .
$$

We readily verify that our system (3.3)-(3.6) admits the energy integral

$$
\frac{1}{2} \int_{0}^{1} \theta_{s}(s, t)^{2} d s+\frac{1}{2} \mu\left|\boldsymbol{r}_{t}(1, t)\right|^{2}=\text { const } .
$$

Note that all the potential energy comes from the rod and all the kinetic energy comes from the end mass. 
That the only time derivatives in the system (3.3)-(3.6) operate on $\boldsymbol{r}(1, t)$ suggests that the only initial conditions for this system should be that

$$
\boldsymbol{r}(1,0) \text { and } \boldsymbol{r}_{t}(1,0) \text { are prescribed. }
$$

Of course, $|\boldsymbol{r}(1,0)|$ should be $\leq 1$ and $\partial_{t}|\boldsymbol{r}(1,0)|$ should be $\leq 0$ if $|\boldsymbol{r}(1,0)|=1$, in consonance with the inextensibility. Our initial-boundary-value problem consists of (3.3)-(3.6), (3.8). Since (3.3) has no time derivatives, $\theta$ evolves through a sequence of equilibrium states. Accordingly, its initial state should be an equilibrium state consistent with (3.5) and (3.8). Since this problem is degenerate, we cannot expect solutions to be regular in a traditional sense. Since the end mass has inertia, we provisionally require that $\boldsymbol{r}(1, \cdot)$ be continuous. Since the rod has no inertia, we do not require that $r$ and $\theta$ be continuous functions of $s$ and $t$, but merely be continuous functions of $s$ for each fixed $t$. (This is the minimal regularity needed to make the mechanics meaningful. This continuity actually ensures that these functions are twice continuously differentiable functions of $s$.)

Now we indicate how to solve our initial-boundary-value problem and describe the striking behavior of solutions. For any given $\lambda, \alpha$ the boundary-value problem

$$
\theta_{s s}(s)+\lambda^{2} \sin (\theta(s)-\alpha)=0, \quad \theta(0)=0=\theta_{s}(1)
$$

has at least one solution (as can be easily shown by the direct methods of the calculus of variations) and typically has a finite number of solutions (the construction of which is described below). Let us denote any such solution of (3.9) by $s \mapsto \tilde{\theta}(s ; \lambda, \alpha)$. Thus if $t \mapsto \lambda(t), \alpha(t)$ are given, then the solutions of (3.3), (3.4) have the form $\theta(s, t)=$ $\tilde{\theta}(s ; \lambda(t), \alpha(t))$.

Let

$$
\psi \equiv \theta-\alpha
$$

Then the resulting version of (3.9a) admits the integral

$$
\frac{1}{2} \psi_{s}^{2}=\lambda^{2}[\cos \psi-\cos \psi(1)]=2 \lambda^{2}\left[\sin ^{2} \frac{1}{2} \psi(1)-\sin ^{2} \frac{1}{2} \psi\right]
$$

which defines the trajectories in the phase portrait, Fig. 3.12, of (3.9a). Candidates for the solutions of the boundary-value problem (3.9) correspond to the trajectories of the form $\mathrm{AB}, \mathrm{CD}, \mathrm{ABCD}, \mathrm{CDAB}, \mathrm{ABCDAB}, \ldots$ in the phase portrait, which must lie on closed orbits encircling the origin. The $(\alpha, \lambda, \theta)$ for which (3.9) has nontrivial solutions lie on two-dimensional solution surfaces $\mathcal{S}_{j}^{ \pm}, j=0,1,2, \ldots$, with $\mathcal{S}_{0}^{+}, \mathcal{S}_{0}^{-}, \mathcal{S}_{1}^{+}, \mathcal{S}_{1}^{-}, \mathcal{S}_{2}^{+}, \ldots$ corresponding to trajectories of the form AB, CD, ABCD, CDAB, ABCDAB,... The subscript $j$ on $\mathcal{S}_{j}^{ \pm}$indicates that $\theta_{s}$ has exactly $j$ zeros in the open interval $(0,1)$ and the superscripts + and - correspond to the sign of $\theta_{s}(0)$. (We apply the same notational scheme to related functions.) Given $\alpha, \lambda$, there is a solution $(\alpha, \lambda, \theta) \in \mathcal{S}_{j}^{ \pm}$if a trajectory of the requisite form can be found so that $s$ varies from 0 to 1 as the trajectory is traversed.

Let us introduce new variables $k, \phi, \beta$ by

$$
\begin{gathered}
k \equiv\left|\sin \frac{1}{2} \psi(1)\right|, \quad k \sin \phi \equiv \sin \frac{1}{2} \psi, \\
\beta \equiv \arcsin \left(\frac{\sin \frac{1}{2} \alpha}{k}\right) \Leftrightarrow \alpha=2 \arcsin (k \sin \beta)
\end{gathered}
$$




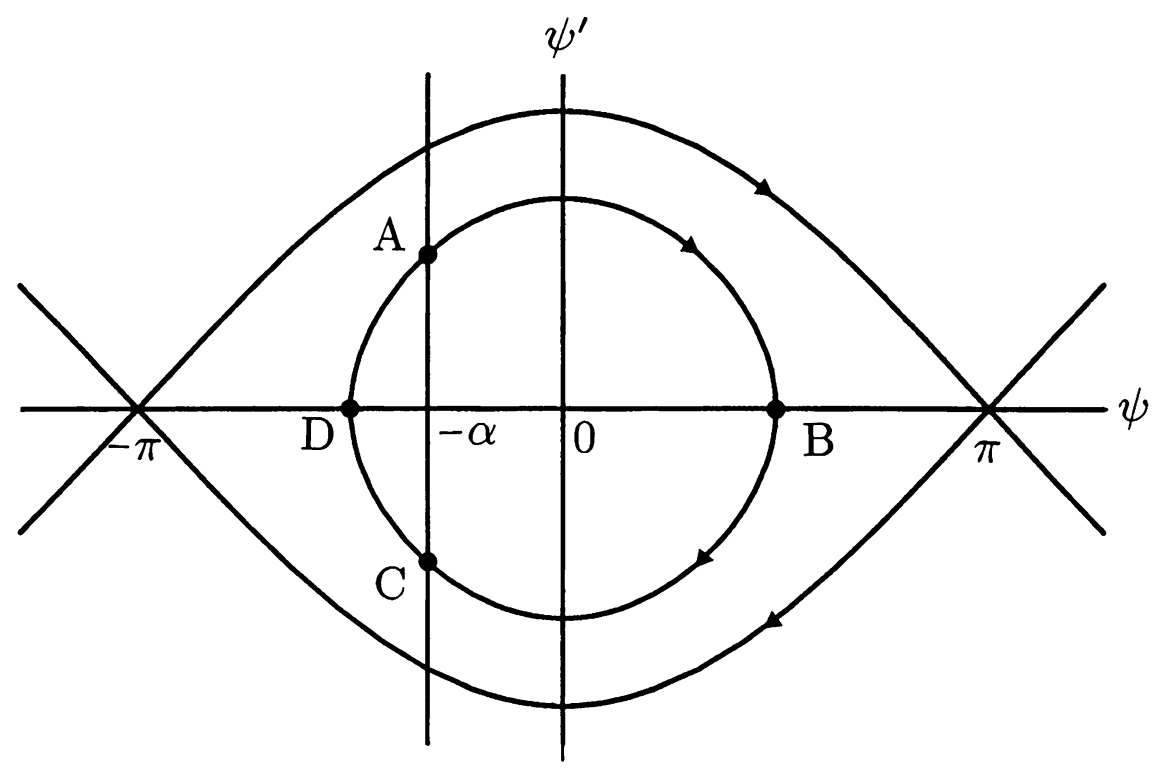

FIG. 3.12. Phase portrait of (3.9) in terms of the variable $\psi=\theta-\alpha$

with $\phi$ and $\beta$ restricted to $\left(-\frac{\pi}{2}, \frac{\pi}{2}\right)$. Then $\mathcal{S}_{j}^{ \pm}$is described in terms of the parameters $k \in[0,1), \beta \in\left(-\frac{\pi}{2}, \frac{\pi}{2}\right)$ by

$$
\begin{gathered}
\alpha=\alpha(\beta, k) \equiv 2 \arcsin (k \sin \beta), \\
\lambda=\lambda_{j}^{ \pm}(\beta, k) \equiv(2 j+1) K(k) \pm F(\beta, k),
\end{gathered}
$$

$$
\sin \frac{1}{2} \psi(s)=k \sin \phi(s)=k \sin \phi_{j}^{ \pm}(s ; \beta, k) \equiv \pm k \operatorname{sn}\left(\lambda_{j}^{ \pm}(\beta, k) s \mp F(\beta, k), k\right)
$$

where $F(\phi, k) \equiv \int_{0}^{\phi}\left(1-k^{2} \sin ^{2} \omega\right)^{-1 / 2} d \omega$ is the incomplete elliptic integral of the first kind with modulus $k$, where $K(k) \equiv F\left(\frac{\pi}{2}, k\right)$ is the corresponding complete elliptic integral, and where $\operatorname{sn}(\cdot, k)$ is the Jacobi elliptic sine-amplitude function with modulus $k$. From (3.14) it follows that

$$
r_{j}^{ \pm}(s ; \beta, k) \equiv x_{j}^{ \pm}(s ; \beta, k) \boldsymbol{k}+y_{j}^{ \pm}(s ; \beta, k) \boldsymbol{i} \equiv \int_{0}^{s}\left[\cos \theta_{j}^{ \pm}(\xi ; \beta, k) \boldsymbol{k}+\sin \theta_{j}^{ \pm}(\xi ; \beta, k) \boldsymbol{i}\right] d \xi
$$

is given by

$$
\begin{aligned}
\cos \alpha(\beta, k) x_{j}^{ \pm}(s ; \beta, k) & +\sin \alpha(\beta, k) y_{j}^{ \pm}(s ; \beta, k) \\
& =-s+\frac{2}{\lambda_{j}^{ \pm}(\beta, k)}\left[E\left(\operatorname{am}\left(\lambda_{j}^{ \pm}(\beta, k) s \mp F(\beta, k)\right), k\right) \pm E(\beta, k)\right], \\
-\sin \alpha(\beta, k) x_{j}^{ \pm}(s ; \beta, k)+ & \cos \alpha(\beta, k) y_{j}^{ \pm}(s ; \beta, k) \\
& =\mp \frac{2 k}{\lambda_{j}^{ \pm}(\beta, k)}\left[\operatorname{cn}\left(\lambda_{j}^{ \pm}(\beta, k) s \mp F(\beta, k), k\right)-\operatorname{cn}(F(\beta, k), k)\right]
\end{aligned}
$$


where the function am is defined by $u=F(\operatorname{am}(u, k), k)$, where $E(\phi, k) \equiv \int_{0}^{\phi}(1-$ $\left.k^{2} \sin \omega\right)^{1 / 2} d \omega$ is the incomplete elliptic integral of the second kind with modulus $k$, and where $\operatorname{cn}(\cdot, k)$ is the Jacobi elliptic cosine-amplitude function with modulus $k$. In particular,

$$
\begin{aligned}
\cos \alpha(\beta, k) x_{j}^{ \pm}(1 ; \beta, k)+\sin \alpha(\beta, k) y_{j}^{ \pm}(1 ; \beta, k) & =-1+2 \frac{(2 j+1) E(k) \pm E(\beta, k)}{(2 j+1) K(k) \pm F(\beta, k)}, \\
-\sin \alpha(\beta, k) x_{j}^{ \pm}(1 ; \beta, k)+\cos \alpha(\beta, k) y_{j}^{ \pm}(1 ; \beta, k) & = \pm \frac{2 k \operatorname{cn}(F(\beta, k), k)}{(2 j+1) K(k) \pm F(\beta, k)}
\end{aligned}
$$

where $E(k) \equiv E\left(\frac{\pi}{2}, k\right)$ is the complete elliptic integral of the second kind. A convenient magnitude of the solution $(3.14 \mathrm{c})$ is

$$
\theta_{j}^{ \pm}(1, \beta, k)=\alpha(\beta, k) \pm 2(-1)^{j} \arcsin k .
$$

Note that the Jacobian of the right-hand sides of $(3.14 \mathrm{a}, \mathrm{b})$ with respect to $k$ and $\beta$ does not have a fixed sign, so that we cannot expect to obtain a smooth inverse of these equations.

Let us illustrate the method of deriving these results for the typical example of $\theta_{1}^{+}$ corresponding to the trajectory $\mathrm{ABCD}$ of Figure 3.12: Let $s_{\mathrm{B}}$ be the amount of the independent variable $s$ used up along the segment AB of Figure 3.12. Then (3.11) and (3.13) imply that

$$
\begin{aligned}
\lambda s & =\frac{1}{2} \int_{-\alpha}^{\psi(s)} \frac{d \psi}{\sqrt{k^{2}-\sin ^{2} \frac{1}{2} \psi}}=\int_{-\beta}^{\phi(s)} \frac{d \phi}{\sqrt{1-k^{2} \sin ^{2} \phi}} \\
& =F(\phi(s), k)+F(\beta, k) \quad \text { for } 0 \leq s \leq s_{\mathbf{B}} \\
\lambda\left(s-s_{\mathbf{B}}\right) & =-\frac{1}{2} \int_{\psi\left(s_{\mathbf{B}}\right)}^{\psi(s)}=\frac{d \psi}{\sqrt{k^{2}-\sin ^{2} \frac{1}{2} \psi}}=-\int_{\pi / 2}^{\phi(s)} \frac{d \phi}{\sqrt{1-k^{2} \sin ^{2} \phi}} \\
& =K(k)-F(\phi(s), k) \text { for } s_{\mathbf{B}} \leq s \leq 1 .
\end{aligned}
$$

System (3.19) yields

$$
\sin \frac{1}{2} \psi_{1}^{+}=k \operatorname{sn}(\lambda s-F(\beta, k), k) .
$$

Setting $s=s_{\mathbf{B}}$ in $(3.19 \mathrm{a})$, setting $s=1$ in $(3.19 \mathrm{~b})$, and adding the resulting equations, we obtain

$$
\lambda=3 K(k)+F(\beta, k) .
$$

(Equation (3.20) merely implies (3.21) modulo $4 K(k)$.)

In Figure 3.22 we show the projections of the solutions sheets $\mathcal{S}_{j}^{ \pm}$for $j=0,1$ onto $(\lambda, \alpha, \theta(1))$-space. We use (3.17) to pull back the surfaces $\mathcal{S}_{j}^{ \pm}$to corresponding surfaces $\mathcal{R}_{j}^{ \pm}$over $(x, y)$-space:

$$
(\beta, k) \mapsto x_{j}^{ \pm}(1 ; \beta, k), y_{j}^{ \pm}(1 ; \beta, k), \theta_{j}^{ \pm}(\cdot ; \beta, k) .
$$

In Figure 3.24 we show the projections of the surfaces $\mathcal{R}_{j}^{ \pm}$onto $(s, y, \theta(1))$-space for $j=0,1$. (These figures were obtained by using Mathematica.) 


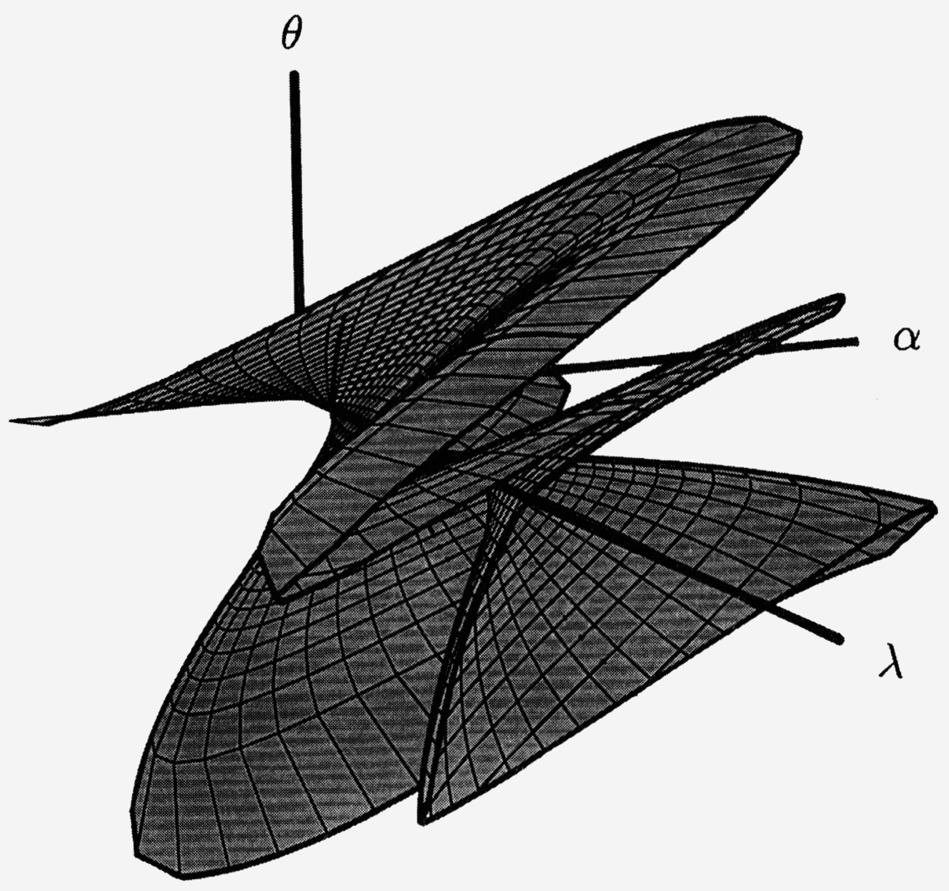

FIG. 3.22. Solution sheets of (3.9)

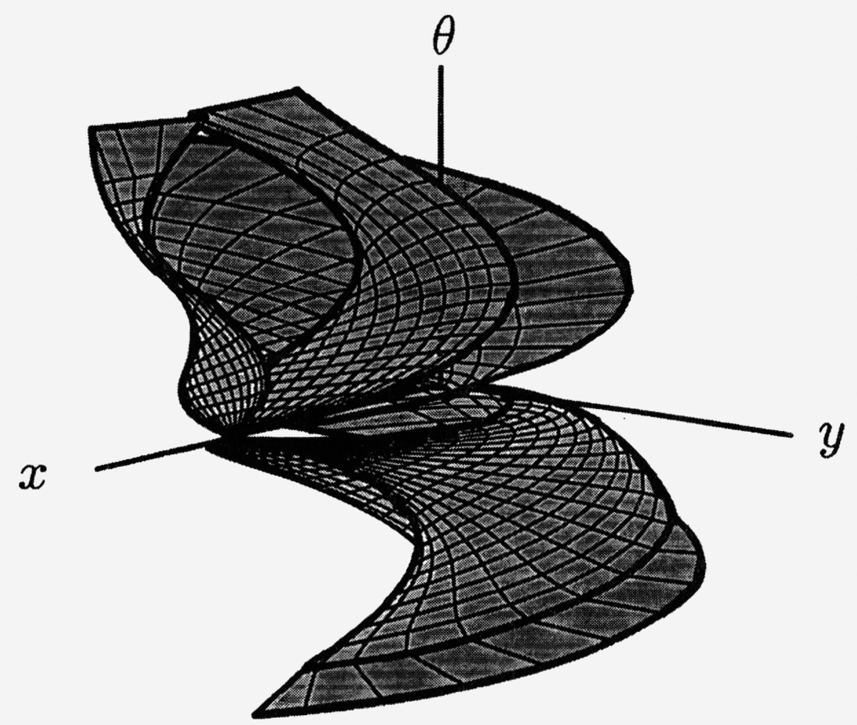

FIG. 3.24. Graphs of $(\beta, k) \mapsto\left(x_{j}^{ \pm}(1 ; \beta, k), y_{j}^{ \pm}(1 ; \beta, k), \theta_{j}^{ \pm}(1 ; \beta, k)\right)$ for $j=0,1$

For detailed information on elliptic functions and integrals see [5]; for applications to other elastica problems, see $[7,10,12]$, among others. 
We now turn to the dynamics of the end mass. For given $\boldsymbol{r}(1, t)$ with $|\boldsymbol{r}(1, t)|<1$ (in conformity with the constraint that $\left|\boldsymbol{r}_{s}\right|=1$ ), given $j$, and given sign + or - , we might be able to solve

$$
\boldsymbol{r}(1, t)=\boldsymbol{r}_{j}^{ \pm}(1 ; \beta, k)
$$

for $\beta, k$ as functions of $\boldsymbol{r}(1, t)$. (If $\boldsymbol{r}(1, t)$ lies on the $\boldsymbol{k}$-axis, then for each $j$ and \pm , Eq. (3.25) has a solution $(\beta, k)$, as is shown by either a bifurcation analysis or a direct computation based on the preceding development.) We denote any such solution by $k_{j}^{ \pm}(\boldsymbol{r}(1, t)), \beta_{j}^{ \pm}(\boldsymbol{r}(1, t))$. (There could be more than one.) The substitution of these expressions into the right-hand sides of $(3.14 \mathrm{a}, \mathrm{b})$ generates corresponding expressions denoted $\alpha_{j}^{ \pm}(\boldsymbol{r}(1, t)), \lambda_{j}^{ \pm}(\boldsymbol{r}(1, t))$. Substituting these into the left-hand side of $(3.6)$, we get an ordinary differential equation for $\boldsymbol{r}(1, \cdot)$ corresponding to the solution sheet $\mathcal{S}_{j}^{ \pm}$:

$$
\mu \boldsymbol{r}_{t t}(1, t)=\lambda_{j}^{ \pm}(\boldsymbol{r}(1, t))^{2}\left[\cos \alpha_{j}^{ \pm}(\boldsymbol{r}(1, t)) \boldsymbol{k}+\sin \alpha_{j}^{ \pm}(\boldsymbol{r}(1, t)) \boldsymbol{i}\right] .
$$

From this ordinary differential equation we can read off the equivalent spring force exerted by the elastica on the end mass when the elastica is moving through equilibrium states on the sheet $\mathcal{S}_{j}^{ \pm}$.

Note that (3.25) is locally solvable for $k$ and $\beta$ when

$$
\partial_{k} x_{j}^{ \pm}(1, \beta, k) \partial_{\beta} y_{j}^{ \pm}(1, \beta, k)-\partial_{\beta} x_{j}^{ \pm}(1, \beta, k) \partial_{k} y_{j}^{ \pm}(1, \beta, k) \neq 0 .
$$

Now we turn to our initial-boundary-value problem. In consonance with the discussion in the paragraph containing (3.8) we prescribe $\boldsymbol{r}(1,0)$ and $\boldsymbol{r}_{t}(1,0)$, and along with these data we choose a solution surface $\mathcal{R}_{j}^{ \pm}$and prescribe a compatible initial configuration on it, i.e., an initial configuration that satisfies

$$
\boldsymbol{r}(1,0)=\boldsymbol{r}_{j}^{ \pm}(1 ; \beta, k) .
$$

(As Fig. 3.24 suggests, there are very few solutions $\theta_{j}^{ \pm}(\cdot ; \beta, k)$ that would satisfy (3.28).) The evolution of the position $\boldsymbol{r}(1, \cdot)$ of the end mass is governed by $(3.26)$ and the initial conditions (3.8) as long as the elastica moves through equilibrium states on the sheet $\mathcal{S}_{j}^{ \pm}$. Now, as Fig. 3.24 shows, the sheet $\mathcal{R}_{j}^{ \pm}$has folds, where this surface is "vertical" above the $(x, y)$-plane. These folds are signalled by the failure of (3.27). What happens when the evolution of $\boldsymbol{r}(1, \cdot)$ brings it to the projection of a fold onto the $(x, y)$-plane, say at time $\tau$ ? We propose the following scenario:

The solution jumps instantaneously to one of the two stable solutions on $\mathcal{R}_{0}^{+} \cup \mathcal{R}_{0}^{-}$ at a point with the same $\boldsymbol{r}(1, \tau)$. We thereby ensure the continuity of $\boldsymbol{r}(1, \cdot)$. By a stable solution we mean one that locally minimizes the energy $\int_{0}^{1} \theta_{s}(s)^{2} d s$ subject to the inextensibility condition, the prescription of $(3.9 \mathrm{~b})$, and the prescription of $\boldsymbol{r}(1, \tau)$. In Figs. 3.22 and 3.24, the stable sheets are those with graphs most remote from the plane of trivial solutions. We have no information to indicate to which of the two stable sheets the solution jumps. Indeed, for a primitive model for the buckling of a rod, Reiss and Matkowsky [13] showed that in the presence of a damping mechanism, the domain of attraction of the stable solutions is exceedingly complicated.

We require initial conditions at time $\tau$ for $\boldsymbol{r}(1, t)$, whose motion is described by (3.26) with $j=0$ and with a choice of \pm . The assumed continuity of $\boldsymbol{r}(1, \cdot)$ says that the initial 
value for it is just $\boldsymbol{r}(1, \tau)$. To get the initial velocity, we appeal to the reduced version of the impulse-momentum law (2.11) in which we substitute (2.42a):

$$
-\int_{\tau-\eta}^{\tau+\eta} \boldsymbol{n}(1, t) d t=\left.\mu\left[\boldsymbol{r}_{t}(1, t)+\boldsymbol{c}_{t}(t)\right]\right|_{t=\tau-\eta} ^{t=\tau+\eta} .
$$

Since our preceding analysis indicates that the corresponding jumps in $\alpha$ and $\lambda$ are finite, we conclude from $(3.29)$ that $\boldsymbol{r}_{t}(1, \cdot)$ is continuous, and we get the desired initial condition for $\boldsymbol{r}_{t}(1, t)$ from this fact. (The angular impulse-momentum law (2.12) yields further information, not needed for our present study. The two laws (2.11), (2.12) imply the conservation of energy (3.7).)

We can now trace the motion on a stable sheet. It can happen that this motion reaches an edge, in which case we surmise that the solution jumps to the other stable sheet; we analyze this jump just as before. Since our construction respects the reduced forms of the impulse-momentum laws, we may regard our solution as a physically natural weak solution.

As an example of this process, suppose that the initial configuration of the elastica is a buckled state, which is of the form $\theta_{j}^{ \pm}(\cdot, k, 0)$ with $j \geq 1$. Based upon experiments with flexible rulers, we might expect that after a short time the elastica vibrates in its lowest mode. Our approach delivers precisely such a conclusion. Indeed, the process by which the shape jumps from a higher buckling mode to the fundamental mode is a version of snap-buckling.

Our approach is reminiscent of applications of catastrophe theory. It differs from the standard applications not only by being global, but more importantly, by having the evolution described by a complete dynamics.

That we cannot handle arbitrary initial data and that we need some dissipation to justify any asymptotics is one of the motivations for treating viscoelastic rods in Sec. 5 . In the next section we show how the ideas used in this section can be generalized for the motion of rods in space.

REMARK. We introduced the parameters $\beta, k$ because they are useful for explicit representations of solutions. When such representations are not available we can work directly with $\alpha, \lambda$, in which case our starting point would be the collection of solutions $\tilde{\theta}(\cdot, \lambda, \alpha)$ of $(3.9)$. In particular, when we can assign nodal properties to solutions by using the phase portrait Fig. 3.12, or more generally, by using a Prüfer transformation, we can label such solutions with subscript $j$ and superscripts \pm , as in (3.14). Suppose that this is the case. As an alternative to describing the motion by (3.26) we can obtain ordinary differential equations for $\lambda, \alpha$ : We let $t \mapsto \lambda_{j}^{ \pm}(t), \alpha_{j}^{ \pm}(t)$ denote the solution of the analog of $(3.25)$ in which $(\beta, k)$ is replaced with $(\lambda, \alpha)$. We substitute $\lambda_{j}^{ \pm}(t), \alpha_{j}^{ \pm}(t)$ into this equation and differentiate it twice with respect to $t$ and then substitute (3.6) into the resulting equation to obtain a system of second-order ordinary differential equations for $t \mapsto \lambda_{j}^{ \pm}(t), \alpha_{j}^{ \pm}(t)$. It can be shown that this system can be locally put into standard form when (3.27) holds.

4. The reduced problem for elastic rods. We now treat the general reduced problem for elastic rods by a straightforward analogy with the treatment of the example 
of Sec. 3. Substituting the integrals (2.42) into the constitutive equations (2.30) we obtain

$$
\mathbf{u}(s, t)=\hat{\mathbf{u}}(\mathbf{m}(s, t), \mathbf{n}(s, t), s), \quad \mathbf{v}(s, t)=\hat{\mathbf{v}}(\mathbf{m}(s, t), \mathbf{n}(s, t), s)
$$

where

$$
\begin{aligned}
m_{k}(s, t) & =\{\boldsymbol{m}(1, t)+[\boldsymbol{r}(1, t)-\boldsymbol{r}(s, t)] \times \boldsymbol{n}(1, t)\} \cdot \boldsymbol{d}_{k}(s, t), \\
n_{k}(s, t) & =\boldsymbol{n}(1, t) \cdot \boldsymbol{d}_{k}(s, t) .
\end{aligned}
$$

Thus (2.3a), (2.4a,b), and (2.9) imply that

$$
\begin{gathered}
\boldsymbol{r}(s, t)=\int_{0}^{s} \hat{v}_{k}(\mathbf{m}(\xi, t), \mathbf{n}(\xi, t), \xi) \boldsymbol{d}_{k}(\xi, t) d \xi \\
\boldsymbol{d}_{k}(s, t)=\boldsymbol{i}_{k}+\int_{0}^{s} \hat{u}_{l}(\mathbf{m}(\xi, t), \mathbf{n}(\xi, t), \xi) \boldsymbol{d}_{l}(\xi, t) \times \boldsymbol{d}_{k}(\xi, t) d \xi
\end{gathered}
$$

where $\mathbf{m}$ and $\mathbf{n}$ are given by (4.2). Let us note that for any given $\boldsymbol{m}(1, t)$ and $\boldsymbol{n}(1, t)$, Eqs. (4.3) and (4.4) are integral equations corresponding to a boundary-value problem for an equilibrium configuration. We know from numerous analyses of buckling and related problems and from the example of Sec. 3 that for each $\boldsymbol{m}(1, t)$ and $\boldsymbol{n}(1, t)$, these equations have at least one solution and they may have several. We denote any such solution by $(s, t) \mapsto \tilde{\boldsymbol{r}}(s, \boldsymbol{m}(1, t), \boldsymbol{n}(1, t)), \tilde{\boldsymbol{d}}_{k}(s, \boldsymbol{m}(1, t), \boldsymbol{n}(1, t))$. We wish to know how the evolution of these solutions is dictated by the evolution of the boundary values $\boldsymbol{r}(1, \cdot)$ and $\boldsymbol{d}_{k}(1, \cdot)$.

Equation (4.3) corresponds to (3.5) and Eq. (4.2) corresponds to (3.3). The variables $\boldsymbol{m}(1, t)$ and $\boldsymbol{n}(1, t)$, correspond to $\lambda(t)$ and $\alpha(t)$ of Sec. 3. The functions $\tilde{\boldsymbol{d}}_{k}$ correspond to $\tilde{\theta}$ introduced after (3.9). We do not introduce parameters corresponding to $k$ and $\beta$ of Sec. 3. See the comments in the last paragraph of that section.

Let us denote any solution $\boldsymbol{m}(1, t), \boldsymbol{n}(1, t)$ of

$$
\tilde{\boldsymbol{r}}(1, \boldsymbol{m}(1, t), \boldsymbol{n}(1, t))=\boldsymbol{r}(1, t), \quad \tilde{\boldsymbol{d}}_{k}(1, \boldsymbol{m}(1, t), \boldsymbol{n}(1, t))=\boldsymbol{d}_{k}(1, t)
$$

by

$$
\boldsymbol{m}(1, t)=\tilde{\boldsymbol{m}}\left(\boldsymbol{r}(1, t), \boldsymbol{d}_{k}(1, t)\right), \quad \boldsymbol{n}(1, t)=\tilde{\boldsymbol{n}}\left(\boldsymbol{r}(1, t), \boldsymbol{d}_{k}(1, t)\right) .
$$

(It is interesting to note that, just as in Sec. 2, the monotonicity condition (2.21) and the growth conditions (2.24) and (2.6) imply that when $\boldsymbol{r}(\cdot, t), \boldsymbol{d}_{k}(\cdot, t)$ are given, system (4.3), (4.4) with $s=1$ can be uniquely solved for $\boldsymbol{m}(1, t)$ and $\boldsymbol{n}(1, t)$ as functions of $\boldsymbol{r}(1, t), \boldsymbol{d}_{k}(1, t)$.) We substitute (4.6) into (2.15) and (2.16) and append (2.3b) to obtain the following multivalued system of ordinary differential equations for $\boldsymbol{r}(1, \cdot), \boldsymbol{d}_{k}(1, \cdot)$, and $\boldsymbol{w}(1, \cdot)$ :

$$
\begin{gathered}
{[\boldsymbol{J}(t) \cdot \boldsymbol{w}(1, t)]_{t}+\mu \boldsymbol{c} \times\left[\boldsymbol{r}_{t t}(1, t)+\boldsymbol{c}_{t t}(t)\right]+\tilde{\boldsymbol{m}}\left(\boldsymbol{r}(1, t), \boldsymbol{d}_{k}(1, t)\right)=\mathbf{0}} \\
\mu\left[\boldsymbol{r}_{t t}(1, t)+\boldsymbol{c}_{t t}(t)\right]+\tilde{\boldsymbol{n}}\left(\boldsymbol{r}(1, t), \boldsymbol{d}_{k}(1, t)\right)=\mathbf{0} \\
\partial_{t} \boldsymbol{d}_{k}(1, t)=\boldsymbol{w}(1, t) \times \boldsymbol{d}_{k}(1, t)
\end{gathered}
$$


We treat this system just as we treated (3.26). In particular, we can construct an analogous theory of catastrophic jumps. From (4.7) and (4.8) we read off the equivalent torsional and longitudinal spring forces exerted by the rod on the rigid body.

For straight, uniform, transversely isotropic rods, the governing equilibrium equations are totally integrable. Their analysis can therefore be reduced to the study of a family of phase portraits parametrized by $\boldsymbol{m}(1, t), \boldsymbol{n}(1, t)$ (cf. [2]). We can accordingly assign nodal properties to the solutions $\tilde{\boldsymbol{r}}, \tilde{\boldsymbol{d}}_{k}$ of $(4.3)$, (4.4) just as we did for the solutions of (3.9).

REMARK. If we do not require that (2.21) hold, then conditions (2.23) or alternatively conditions (2.24), (2.26) ensure that (2.27) can be solved for $\mathbf{u}$ and $\mathbf{v}$, but the solution need not be unique. We may then regard (2.28) as multivalued. Since this multivaluedness merely adds to that already present, we could carry out the entire development of this section without (2.21).

\section{The reduced problem for viscoelastic rods.}

The degenerate initial-value problem. In this section we first convert the reduced problem for viscoelastic rods to an equivalent degenerate initial-value problem, which we show has classical solutions defined for all positive time. Substituting the integrals (2.42) into the constitutive equations (2.39) we obtain

$$
\begin{aligned}
& \mathbf{u}_{t}(s, t)=\dot{\mathbf{u}}^{\sharp}(\mathbf{u}(s, t), \mathbf{v}(s, t), \mathbf{m}(s, t), \mathbf{n}(s, t), s), \\
& \mathbf{v}_{t}(s, t)=\dot{\mathbf{v}}^{\sharp}(\mathbf{u}(s, t), \mathbf{v}(s, t), \mathbf{m}(s, t), \mathbf{n}(s, t), s)
\end{aligned}
$$

where

$$
\begin{aligned}
m_{k}(s, t) & =\{\boldsymbol{m}(1, t)+[\boldsymbol{r}(1, t)-\boldsymbol{r}(s, t)] \times \boldsymbol{n}(1, t)\} \cdot \boldsymbol{d}_{k}(s, t), \\
n_{k}(s, t) & =\boldsymbol{n}(1, t) \cdot \boldsymbol{d}_{k}(s, t) .
\end{aligned}
$$

From (2.4b) and (2.5) we obtain $\boldsymbol{r}_{s t}=\left(\partial_{t} v_{k}\right) \boldsymbol{d}_{k}+\boldsymbol{w} \times \boldsymbol{r}_{s}$. Thus (2.9) and (5.1) imply that

$$
\begin{aligned}
\boldsymbol{r}_{t}(s, t)= & \int_{0}^{s}\left[\dot{v}_{k}^{\sharp}(\mathbf{u}(\xi, t), \mathbf{v}(\xi, t), \mathbf{m}(\xi, t), \mathbf{n}(\xi, t), s) \boldsymbol{d}_{k}(\xi, t)\right. \\
& \left.\quad+\boldsymbol{w}(\xi, t) \times v_{k}(\xi, t) \boldsymbol{d}_{k}(\xi, t)\right] d \xi \\
= & \int_{0}^{s}\left\{\dot{v}_{k}^{\sharp}(\mathbf{u}(\xi, t), \mathbf{v}(\xi, t), \mathbf{m}(\xi, t), \mathbf{n}(\xi, t), s) \boldsymbol{d}_{k}(\xi, t)\right. \\
& \left.\quad+\dot{u}_{k}^{\sharp}(\mathbf{u}(\xi, t), \mathbf{v}(\xi, t), \mathbf{m}(\xi, t), \mathbf{n}(\xi, t), s) \boldsymbol{d}_{k}(\xi, t) \times[\boldsymbol{r}(s, t)-\boldsymbol{r}(\xi, t)]\right\} d \xi
\end{aligned}
$$

where

$$
\boldsymbol{w}(s, t)=\int_{0}^{s} \dot{u}_{k}^{\sharp}(\mathbf{u}(\xi, t), \mathbf{v}(\xi, t), \mathbf{m}(\xi, t), \mathbf{n}(\xi, t), s) \boldsymbol{d}_{k}(\xi, t) d \xi .
$$

Likewise (2.3b) yields

$$
\partial_{t} \boldsymbol{d}_{k}=\boldsymbol{w} \times \boldsymbol{d}_{k}
$$

with $\boldsymbol{w}$ given by (5.4).

If $\boldsymbol{m}(1, \cdot)$ and $\boldsymbol{n}(1, \cdot)$ were known, then (5.1), (5.3), (5.5) would constitute a system of ordinary differential equations for the family $\left\{\mathbf{u}(s, \cdot), \mathbf{v}(s, \cdot), \boldsymbol{r}(s, \cdot), \boldsymbol{d}_{k}(s, \cdot): s \in[0,1]\right\}$. That this family of functions is parametrized by $s$ is just the remnant of the full partial 
differential equations valid for $\varepsilon \neq 0$. We now show how to augment this system with ordinary differential equations for $\boldsymbol{m}(1, \cdot)$ and $\boldsymbol{n}(1, \cdot)$.

From (2.15)-(2.17), (5.1), and (5.3) we obtain

$$
\begin{gathered}
-\frac{\boldsymbol{n}(1, t)}{\mu}-\boldsymbol{c}_{t t}(t)=\boldsymbol{r}_{t t}(1, t) \\
=\int_{0}^{1}\left\{\left[\frac{\partial \dot{v}_{k}^{\sharp}}{\partial \mathbf{u}} \cdot \dot{\mathbf{u}}^{\sharp}+\frac{\partial \dot{v}_{k}^{\sharp}}{\partial \mathbf{v}} \cdot \dot{\mathbf{v}}^{\sharp}+\frac{\partial \dot{v}_{k}^{\sharp}}{\partial \mathbf{m}} \cdot \mathbf{m}_{t}+\frac{\partial \dot{v}_{k}^{\sharp}}{\partial \mathbf{n}} \cdot \mathbf{n}_{t}\right] \boldsymbol{d}_{k}\right. \\
\left.\quad+2 \dot{v}_{k}^{\sharp} \boldsymbol{w} \times \boldsymbol{d}_{k}+v_{k}\left[\boldsymbol{w}_{t} \times \boldsymbol{d}_{k}+\boldsymbol{w} \times\left(\boldsymbol{w} \times \boldsymbol{d}_{k}\right)\right]\right\} d s, \\
\boldsymbol{J}(t)^{-1} \cdot\{-\boldsymbol{m}(1, t)+\boldsymbol{c}(t) \times \boldsymbol{n}(1, t)-\boldsymbol{w}(1, t) \times[\boldsymbol{J}(t) \cdot \boldsymbol{w}(1, t)]\}=\boldsymbol{w}_{t}(1, t) \\
=\int_{0}^{1}\left\{\left[\frac{\partial \dot{u}_{k}^{\sharp}}{\partial \mathbf{u}} \cdot \dot{\mathbf{u}}^{\sharp}+\frac{\partial \dot{u}_{k}^{\sharp}}{\partial \mathbf{v}} \cdot \dot{\mathbf{v}}^{\sharp}+\frac{\partial \dot{u}_{k}^{\sharp}}{\partial \mathbf{m}} \cdot \mathbf{m}_{t}+\frac{\partial \dot{u}_{k}^{\sharp}}{\partial \mathbf{n}} \cdot \mathbf{n}_{t}\right] \boldsymbol{d}_{k}+\dot{u}_{k}^{\sharp} \boldsymbol{w} \times \boldsymbol{d}_{k}\right\} d s
\end{gathered}
$$

where

$$
\begin{aligned}
\partial_{t} m_{k}(s, t)= & \left\{\boldsymbol{m}_{t}(1, t)+\left[\boldsymbol{d}_{k}(s, t) \times(\boldsymbol{r}(1, t)-\boldsymbol{r}(s, t))\right] \cdot \boldsymbol{n}_{t}(1, t)\right. \\
& \left.+\left[\boldsymbol{r}_{t}(1, t)-\boldsymbol{r}_{t}(s, t)\right] \times \boldsymbol{n}(1, t)\right\} \cdot \boldsymbol{d}_{k}(s, t)+m_{k}(s, t) \boldsymbol{w}(s, t) \times \boldsymbol{d}_{k}(s, t), \\
\partial_{t} n_{k}(s, t)= & \boldsymbol{n}_{t}(1, t) \cdot \boldsymbol{d}_{k}(s, t)+n_{k}(s, t) \boldsymbol{w}(s, t) \times \boldsymbol{d}_{k}(s, t),
\end{aligned}
$$

where (5.2)-(5.4) hold, and where the $\boldsymbol{w}_{t}$ in (5.6) is given by the integral (5.7) with its upper limit of integration 1 replaced with $s$.

We now show that we can solve (5.6) and (5.7) uniquely for $\boldsymbol{m}_{t}(1, t)$ and $\boldsymbol{n}_{t}(1, t)$ in terms of $\boldsymbol{m}(1, t), \boldsymbol{n}(1, t)$, and the other variables that appear in (5.1), (5.3), and (5.5) by showing that the matrix of coefficients of $\boldsymbol{m}_{t}(1, t)$ and $\boldsymbol{n}_{t}(1, t)$ in $(5.7)$ and (5.6) is positive-definite. The terms containing $\boldsymbol{m}_{t}(1, t)$ and $\boldsymbol{n}_{t}(1, t)$ in $(5.7)$ and $(5.6)$ are

$$
\begin{aligned}
\int_{0}^{1} & \left\{\frac{\partial \dot{u}_{k}^{\sharp}}{\partial m_{l}} \boldsymbol{d}_{l} \cdot \boldsymbol{m}_{t}(1, t)+\left[\frac{\partial \dot{u}_{k}^{\sharp}}{\partial m_{l}} \boldsymbol{q}_{l}+\frac{\partial \dot{u}_{k}^{\sharp}}{\partial n_{l}} \boldsymbol{d}_{l}\right] \cdot \boldsymbol{n}_{t}(1, t)\right\} \boldsymbol{d}_{k} d s \\
& \equiv \boldsymbol{A}(t) \cdot \boldsymbol{m}_{t}(1, t)+\boldsymbol{B}(t) \cdot \boldsymbol{n}_{t}(1, t), \\
\int_{0}^{1} & \left\{\frac{\partial \dot{v}_{k}^{\sharp}}{\partial m_{l}} \boldsymbol{d}_{l} \cdot \boldsymbol{m}_{t}(1, t)+\left[\frac{\partial \dot{v}_{k}^{\sharp}}{\partial m_{l}} \boldsymbol{q}_{l}+\frac{\partial \dot{u}_{k}^{\sharp}}{\partial n_{l}} \boldsymbol{d}_{l}\right] \cdot \boldsymbol{n}_{t}(1, t)\right\} \boldsymbol{d}_{k} d s \\
& \equiv \boldsymbol{C}(t) \cdot \boldsymbol{m}_{t}(1, t)+\boldsymbol{D}(t) \cdot \boldsymbol{n}_{t}(1, t),
\end{aligned}
$$

where $\boldsymbol{q}_{l}(s, t) \equiv \boldsymbol{d}_{l}(s, t) \times[\boldsymbol{r}(1, t)-\boldsymbol{r}(s, t)]$. Note that the first line of (5.10) comes from (5.7) and the second from (5.6).

Let $\boldsymbol{a}$ and $\boldsymbol{b}$ be constant vectors, and set

$$
a_{k}(s, t) \equiv \boldsymbol{a} \cdot \boldsymbol{d}_{k}(s, t), \quad b_{k}(s, t) \equiv \boldsymbol{b} \cdot \boldsymbol{d}_{k}(s, t), \quad p_{k}(s, t) \equiv a_{k}(s, t)+\boldsymbol{b} \cdot \boldsymbol{q}_{k}(s, t)
$$


The quadratic form of the coefficients of $\boldsymbol{m}_{t}(1, t)$ and $\boldsymbol{n}_{t}(1, t)$ in (5.7) and (5.6) saturated with vectors $\boldsymbol{a}$ and $\boldsymbol{b}$ is

$$
\begin{aligned}
& \boldsymbol{a} \cdot \boldsymbol{A} \cdot \boldsymbol{a}+\boldsymbol{a} \cdot \boldsymbol{B} \cdot \boldsymbol{b}+\boldsymbol{b} \cdot \boldsymbol{C} \cdot \boldsymbol{a}+\boldsymbol{b} \cdot \boldsymbol{D} \cdot \boldsymbol{b} \\
&=\int_{0}^{1}\left\{p_{k} \frac{\partial \dot{u}_{k}^{\sharp}}{\partial m_{l}} p_{l}+p_{k} \frac{\partial \dot{u}_{k}^{\sharp}}{\partial n_{l}} b_{l}+b_{k} \frac{\partial \dot{v}_{k}^{\sharp}}{\partial m_{l}} p_{l}+b_{k} \frac{\partial \dot{v}_{k}^{\sharp}}{\partial n_{l}} b_{l}\right\} d s,
\end{aligned}
$$

which is positive-definite by (2.38). We can accordingly solve (5.6) and (5.7) for $\boldsymbol{m}_{t}(1, t)$ and $\boldsymbol{n}_{t}(1, t)$ and adjoin the resulting equations to (5.1), (5.3), (5.5) to obtain a formally determinate system of ordinary differential equations.

REMARK. Note that (2.38) enables us to show that the matrix of coefficients in (5.10) is positive-definite because the utility of the positive-definiteness of (2.8) is not diminished by the presence of integrals in (5.10). Although we require only the nonsingularity of the matrix of coefficients in (5.10), it would not be easy to weaken (2.8) and still be able to handle the integrals.

\section{Conditions ensuring that in the reduced problem for viscoelastic rods} the motion of the rigid body is governed by ordinary differential equations. For purely longitudinal motions we take $\boldsymbol{r}(s, t)=r(s, t) \boldsymbol{k}$. Then (2.42a) and (2.20) yield an equation of the form

$$
\hat{n}\left(r_{s}(s, t), r_{s t}(s, t), s\right)=\hat{n}\left(r_{s}(1, t), r_{s t}(1, t), 1\right) .
$$

If $\hat{n}$ is independent of $s$, then (6.1) admits a solution of the form

$$
r_{s}(s, t)=r_{s}(1, t) \quad \text { so that } r(s, t)=s r_{s}(1, t) \quad \text { and } \quad r(1, t)=r_{s}(1, t) .
$$

If the initial conditions are compatible with (6.2), then the analog of (2.15) (with $\boldsymbol{c}=\mathbf{0}$ ) delivers an ordinary differential equation for the end mass:

$$
\mu r_{t t}(1, t)+\hat{n}\left(r(1, t), r_{t}(1, t)\right)=0 .
$$

For other initial conditions, however, the motion of the end mass is generally not governed by an ordinary differential equation (as shown in [1]), so that memory effects enter.

We now study conditions under which the reduced motion of a rigid body attached to a viscoelastic rod is governed by ordinary differential equations. When it is, we can identify an equivalent set of spring and frictional forces. If we substitute the constitutive equations (2.20) into the boundary conditions $(2.15),(2.16)$, we obtain equations that fail to be ordinary differential equations for $\boldsymbol{r}(1, \cdot), \boldsymbol{d}_{k}(1, \cdot)$ because they involve $\mathbf{u}(1, \cdot), \mathbf{v}(1, \cdot)$, i.e., they involve the $s$-derivatives $\boldsymbol{r}_{s}(1, \cdot), \partial_{s} \boldsymbol{d}_{k}(1, \cdot)$. To follow the procedure in the preceding paragraph, we insert (2.20) into (2.42) and ask when we can "solve" the resulting system for $\mathbf{u}(\cdot, t), \ldots$ in such a way that we can express $\mathbf{u}(1, t), \ldots$ in terms of

$$
\boldsymbol{r}(1, t), \boldsymbol{r}_{t}(1, t), \boldsymbol{d}_{k}(1, t), \boldsymbol{w}(1, t)
$$

We begin by showing that in contrast to the case for elastic rods, the reduced problem cannot be governed by ordinary differential equations when the rod undergoes a flexural motion, i.e., a motion with the property that for each fixed $t$, the direction of $\boldsymbol{r}(s, t)$ varies with $s$. For simplicity of exposition we restrict our attention to rods whose natural 
reference configuration is straight and prismatic, so that $\mathbf{r}_{s}$ and $\boldsymbol{d}_{k}$ are constant in this configuration.

For this purpose, we consider a 1-parameter family of initial data:

$$
\begin{array}{llrl}
\boldsymbol{r}(s, 0) & =s \boldsymbol{i}_{3}+\eta \overline{\boldsymbol{r}}(s), & & \boldsymbol{r}_{t}(s, 0)=\eta \overline{\boldsymbol{p}}(s), \\
\boldsymbol{u}(s, 0)=\eta \overline{\boldsymbol{u}}(s), & \boldsymbol{w}(s, 0)=\eta \overline{\boldsymbol{w}}(s) .
\end{array}
$$

For $\eta=0$, the solution of the reduced problem, which is unique by Sec. 7 , is just the reference configuration:

$$
\boldsymbol{r}(s, t)=s \boldsymbol{i}_{3}, \quad \boldsymbol{d}_{k}(s, t)=\boldsymbol{i}_{k}
$$

Now if the reduced problem subject to initial conditions (6.5) is governed by ordinary differential equations, then so is its derivative with respect to $\eta$ at $\eta=0$; this derivative is just the linearization of the reduced problem about the state (6.6). We now show that (except in one peculiar case) this linearized problem cannot be governed by ordinary differential equations when there is flexure.

In this treatment of the linearization, we adhere to the convention that $\mathbf{y}=\left(\boldsymbol{y} \cdot \boldsymbol{i}_{1}\right.$, $\left.\boldsymbol{y} \cdot \boldsymbol{i}_{2}, \boldsymbol{y} \cdot \boldsymbol{i}_{3}\right)$ is the triple of components of $\boldsymbol{y}$ with respect to the Cartesian basis $\left\{\boldsymbol{i}_{k}\right\}$. In particular, $\mathbf{i}_{3}=(0,0,1)$.

If $(s, t) \mapsto \boldsymbol{u}(s, t ; \eta)$ is one of the functions constituting the solution of the reduced problem subject to $(6.4)$, then we set $\delta \boldsymbol{u}(s, t) \equiv \frac{\partial u}{\partial \eta}(s, t ; 0)$. We denote

$$
\frac{\partial \mathbf{m}^{0}}{\partial \mathbf{u}}(s) \equiv \frac{\partial \hat{\mathbf{m}}}{\partial \mathbf{u}}\left(\mathbf{0}, \mathbf{i}_{3}, \mathbf{0}, \mathbf{0}, s\right)
$$

etc. We set

$$
\delta \boldsymbol{d}_{k}(s, t)=\boldsymbol{z}(s, t) \times \boldsymbol{i}_{k}
$$

with $\boldsymbol{z}(0, t)=\mathbf{0}$ by $(2.9 \mathrm{~b})$. In analogy with (2.3)-(2.5) we have

$$
\mathbf{z}_{s}=\delta \mathbf{u} .
$$

We define $\overline{\mathbf{z}}(s)=\int_{0}^{s} \overline{\mathbf{u}}(\xi) d \xi$. By differentiating (2.4b) with respect to $\eta$ and setting $\eta=0$, we get

$$
\delta \boldsymbol{r}_{s}=\delta v_{k} \boldsymbol{i}_{k}+\boldsymbol{z} \times \boldsymbol{i}_{3} \quad \text { or, equivalently, } \quad \delta \mathbf{r}_{s}=\delta \mathbf{v}+\mathbf{z} \times \mathbf{i}_{3} .
$$

Thus (6.5) implies that $\delta \mathbf{v}(s, 0)=\overline{\mathbf{r}}_{s}(s)-\overline{\mathbf{z}} \times \mathbf{i}_{3}$.

We substitute (2.20) into (2.42) and linearize the resulting system to obtain

$$
\begin{aligned}
\mathbf{A}(s) & \cdot\left(\begin{array}{l}
\delta \mathbf{u}(s, t) \\
\delta \mathbf{v}(s, t)
\end{array}\right)_{t}+\mathbf{B}(s) \cdot\left(\begin{array}{l}
\delta \mathbf{u}(s, t) \\
\delta \mathbf{v}(s, t)
\end{array}\right) \\
& =\mathbf{A}(s) \cdot \mathbf{L}(s) \cdot \mathbf{A}(1)^{-1} \cdot\left[\mathbf{A}(1) \cdot\left(\begin{array}{l}
\delta \mathbf{u}(1, t) \\
\delta \mathbf{v}(1, t)
\end{array}\right)_{t}+\mathbf{B}(1) \cdot\left(\begin{array}{l}
\delta \mathbf{u}(1, t) \\
\delta \mathbf{v}(1, t)
\end{array}\right)\right]
\end{aligned}
$$

where

$$
\mathbf{A}(s) \equiv \frac{\partial\left(\mathbf{m}^{0}, \mathbf{n}^{0}\right)}{\partial(\dot{\mathbf{u}}, \dot{\mathbf{v}})}(s), \quad \mathbf{B}(s) \equiv \frac{\partial\left(\mathbf{m}^{0}, \mathbf{v}^{0}\right)}{\partial(\mathbf{u}, \mathbf{v})}(s)
$$




$$
\mathbf{A}(s) \cdot \mathbf{L}(s) \cdot \mathbf{A}(1)^{-1} \equiv \mathbf{I}+(1-s)\left(\begin{array}{cc}
\mathbf{O}_{3} & \mathbf{K}_{3} \\
\mathbf{O}_{3} & \mathbf{O}_{3}
\end{array}\right), \quad \mathbf{K}_{3}=\left(\begin{array}{ccc}
0 & -1 & 0 \\
1 & 0 & 0 \\
0 & 0 & 0
\end{array}\right)
$$

$\mathbf{I}$ is the $6 \times 6$ identity matrix, and $\mathbf{O}_{3}$ is the $3 \times 3$ zero matrix. Let $\mathbf{C}(s) \equiv-\mathbf{A}(s)^{-1} \cdot \mathbf{B}(s)$. Then we can write $(6.10 \mathrm{a})$ as

$$
\begin{aligned}
& {\left[\exp (-\mathbf{C}(s) t) \cdot\left(\begin{array}{l}
\delta \mathbf{u}(s, t) \\
\delta \mathbf{v}(s, t)
\end{array}\right)\right]_{t}} \\
& \quad=\exp (-\mathbf{C}(s) t) \cdot \mathbf{L}(s) \cdot \exp (\mathbf{C}(1) t) \cdot\left[\exp (-\mathbf{C}(1) t) \cdot\left(\begin{array}{l}
\delta \mathbf{u}(1, t) \\
\delta \mathbf{v}(1, t)
\end{array}\right)\right]_{t}
\end{aligned}
$$

We integrate $(6.10 \mathrm{~d})$ to get

$$
\begin{aligned}
\left(\begin{array}{l}
\delta \mathbf{u}(s, t) \\
\delta \mathbf{v}(s, t)
\end{array}\right)=\mathbf{L}(s) & \cdot\left(\begin{array}{l}
\delta \mathbf{u}(1, t) \\
\delta \mathbf{v}(1, t)
\end{array}\right) \\
& +\exp (\mathbf{C}(s) t) \cdot\left[\left(\begin{array}{c}
\overline{\mathbf{u}}(s) \\
\overline{\mathbf{r}}_{s}(s)-\overline{\mathbf{z}}(s) \times \mathbf{i}_{3}
\end{array}\right)-\mathbf{L}(s) \cdot\left(\begin{array}{c}
\overline{\mathbf{u}}(1) \\
\overline{\mathbf{r}}_{s}(1)-\overline{\mathbf{z}}(1) \times \mathbf{i}_{3}
\end{array}\right)\right] \\
& +\int_{0}^{t} \exp (\mathbf{C}(s)(t-\tau))[\mathbf{C}(s) \cdot \mathbf{L}(s)-\mathbf{L}(s) \cdot \mathbf{C}(1)] \cdot\left(\begin{array}{l}
\delta \mathbf{u}(1, \tau) \\
\delta \mathbf{v}(1, \tau)
\end{array}\right) d \tau
\end{aligned}
$$

From $(6.8 \mathrm{~b}),(6.9)$, and $(6.11)$ we obtain

$$
\begin{aligned}
& \left(\begin{array}{c}
\mathbf{z}(s, t) \\
\delta \mathbf{r}(s, t)+\mathbf{i}_{3} \times \int_{0}^{s} \mathbf{z}(\xi, t) d \xi
\end{array}\right) \\
& =\int_{0}^{s} \mathbf{L}(\xi) d \xi \cdot\left(\begin{array}{l}
\delta \mathbf{u}(1, t) \\
\delta \mathbf{v}(1, t)
\end{array}\right) \\
& \quad+\int_{0}^{s} \exp (\mathbf{C}(\xi) t) \cdot\left[\left(\begin{array}{c}
\overline{\mathbf{u}}(\xi) \\
\overline{\mathbf{r}}_{s}(\xi)-\overline{\mathbf{z}}(\xi) \times \mathbf{i}_{3}
\end{array}\right)-\mathbf{L}(\xi) \cdot\left(\begin{array}{c}
\overline{\mathbf{u}}(1) \\
\mathbf{\mathbf { r }}_{s}(1)-\overline{\mathbf{z}}(1) \times \mathbf{i}_{3}
\end{array}\right)\right] d \xi \\
& \quad+\int_{0}^{t} \int_{0}^{s} \exp (\mathbf{C}(\xi)(t-\tau)) \cdot[\mathbf{C}(\xi) \cdot \mathbf{L}(\xi)-\mathbf{L}(\xi) \cdot \mathbf{C}(1)] \cdot\left(\begin{array}{c}
\delta \mathbf{u}(1, \tau) \\
\delta \mathbf{v}(1, \tau)
\end{array}\right) d \xi d \tau
\end{aligned}
$$

To isolate $\mathbf{z}$ from $\delta \mathbf{r}$, we compute $\int_{0}^{1} \mathbf{z}(s, t) d s$ by integrating (6.12) with respect to $s$ over $[0,1]$. Whence

$$
\left(\begin{array}{c}
\mathbf{0} \\
\mathbf{i}_{3} \times \int_{0}^{1} \mathbf{z}(\xi, t) d \xi
\end{array}\right)=\left(\begin{array}{ll}
\mathbf{O}_{3} & \mathbf{O}_{3} \\
\mathbf{K}_{3} & \mathbf{O}_{3}
\end{array}\right) \cdot \int_{0}^{1}(1-s) \mathbf{f}(s, t) d s
$$


where $\int_{0}^{s} \mathbf{f}(\xi, t) d \xi$ denotes the right-hand side of (6.12). Substituting (6.13) into (6.12) evaluated at $s=1$, we obtain

$$
\begin{aligned}
& \left(\begin{array}{c}
\mathbf{z}(1, t) \\
\delta \mathbf{r}(1, t)
\end{array}\right) \\
& \quad=\int_{0}^{1} \mathbf{M}(s) \cdot \mathbf{L}(s) d s \cdot\left(\begin{array}{c}
\delta \mathbf{u}(1, t) \\
\delta \mathbf{v}(1, t)
\end{array}\right) \\
& \quad+\int_{0}^{1} \mathbf{M}(s) \cdot \exp (\mathbf{C}(s) t) \cdot\left[\left(\begin{array}{c}
\overline{\mathbf{u}}(s) \\
\overline{\mathbf{r}}_{s}(s)-\overline{\mathbf{z}}(s) \times \mathbf{i}_{3}
\end{array}\right)-\mathbf{L}(s) \cdot\left(\begin{array}{c}
\overline{\mathbf{u}}(1) \\
\overline{\mathbf{r}}_{s}(1)-\overline{\mathbf{z}}(1) \times \mathbf{i}_{3}
\end{array}\right)\right] d s \\
& \quad+\int_{0}^{t} \int_{0}^{1} \mathbf{M}(s) \cdot \exp (\mathbf{C}(s)(t-\tau)) \cdot[\mathbf{C}(s) \cdot \mathbf{L}(s)-\mathbf{L}(s) \cdot \mathbf{C}(1)] \cdot\left(\begin{array}{l}
\delta \mathbf{u}(1, \tau) \\
\delta \mathbf{v}(1, \tau)
\end{array}\right) d s d \tau
\end{aligned}
$$

where

$$
\mathbf{M}(s) \equiv \mathbf{I}-(1-s)\left(\begin{array}{ll}
\mathbf{O}_{3} & \mathbf{O}_{3} \\
\mathbf{K}_{3} & \mathbf{O}_{3}
\end{array}\right)
$$

Equation (6.14a) is a convolution equation to be solved for $\delta \mathbf{u}(1, \cdot), \delta \mathbf{v}(1, \cdot)$ in terms of $\mathbf{z}(1, \cdot), \mathbf{r}(1, \cdot)$. (The first term on the right-hand side of $(6.14 \mathrm{a})$ may be regarded as convolved with the Dirac delta.) If

$$
\mathbf{C}(s) \cdot \mathbf{L}(s)=\mathbf{L}(s) \cdot \mathbf{C}(1) \quad \forall s,
$$

then clearly (6.14) gives $\delta \mathbf{u}(1, t), \delta \mathbf{v}(1, t)$ as functions of $\mathbf{z}(1, t), \mathbf{r}(1, t)$, so that the reduced linearized problem is governed by ordinary differential equations. These ordinary differential equations are autonomous (as is appropriate if this reduced problem is to be regarded as an equivalent spring-dashpot system) provided that the second integral on the right-hand side of (6.14a), which is the transient, is zero. The natural sufficient condition for this autonomy is that the initial conditions have the special form causing the bracketed expression in this integral to vanish. If the last integral of (6.14a) does not vanish, then the unique solution of (6.14a) would be expressed as a nontrivial convolution with the left-hand side of (6.14a) (see Zemanian [16, Chap. 9]), so that the response of the reduced system would depend on the past history of the motion of the end-body.

The most natural way to ensure (6.15) is to take $\mathbf{C}$ to be constant, which would be a consequence of the independence of the constitutive functions on $s$, or more generally, a consequence of the proportionality of the viscosity matrix $\mathbf{A}$ to the elasticity matrix $\mathbf{B}$, and to take $\mathbf{L}=\mathbf{I}$ to ensure the commutativity in (6.15) when $\mathbf{C}$ does not ensure it. This last condition means that the linearization of

$$
[\boldsymbol{r}(1, t)-\boldsymbol{r}(s, t)] \times \boldsymbol{n}(1, t)=\mathbf{0}
$$

is satisfied, so that there is no flexure. If $\mathbf{L} \neq \mathbf{I}$, then the constitutive equations would have to have a very special form for (6.15) to hold. Such a form would correspond to a very peculiar tapering (cf. [1]). Thus, one of the few natural circumstances under which 
(6.15) holds without (6.16) is that $\mathbf{C}$ is a constant multiple of $\mathbf{I}$. We remark that further perturbations would produce more restrictions on the data necessary to ensure that the reduced problem is governed by ordinary differential equations.

We now seek conditions sufficient to ensure that the nonlinear reduced problem is governed by ordinary differential equations. In view of the necessary conditions we have just obtained, we exclude flexural motions by restricting our attention to motions for which (6.16) holds and to constitutive functions that are independent of $s$. It is shown in [2] that if the constitutive functions for a rod are independent of $s$, then the reference configuration must be of a special form. For simplicity, let us again assume that the rod is initially straight and prismatic, which is one such special form.

Let $\mathbf{D}(s, t)$ be the rotation matrix with components

$$
D_{k l}(s, t)=\boldsymbol{d}_{k}(s, t) \cdot \boldsymbol{d}_{1}(1, t)
$$

Then (6.16) reduces $(2.20)$ and $(2.42)$ to

$$
\begin{gathered}
\hat{\mathbf{m}}\left(\mathbf{u}(s, t), \mathbf{v}(s, t), \mathbf{u}_{t}(s, t), \mathbf{v}_{t}(s, t)\right)=\mathbf{D}(s, t) \cdot \hat{\mathbf{m}}\left(\mathbf{u}(1, t), \mathbf{v}(1, t), \mathbf{u}_{t}(1, t), \mathbf{v}_{t}(1, t)\right), \\
\hat{\mathbf{n}}\left(\mathbf{u}(s, t), \mathbf{v}(s, t), \mathbf{u}_{t}(s, t), \mathbf{v}_{t}(s, t)\right)=\mathbf{D}(s, t) \cdot \hat{\mathbf{n}}\left(\mathbf{u}(1, t), \mathbf{v}(1, t), \mathbf{u}_{t}(1, t), \mathbf{v}_{t}(1, t)\right)
\end{gathered}
$$

Let us first treat the case in which $\mathbf{D}=\mathbf{I}$ or, equivalently, $\boldsymbol{u}=\mathbf{0}$, so that $\boldsymbol{d}_{k}(s, t)=\boldsymbol{i}_{k}$, whence every cross section is perpendicular to the $i_{3}$-axis and does not rotate about it. These motions consist of elongations and shears. In this case, (6.18) admits the solution $\mathbf{v}(s, t)=\mathbf{v}(1, t)$. By $(2.4 \mathrm{~b})$ we get $\boldsymbol{r}(s, t)=s v_{k}(1, t) \boldsymbol{i}_{k}=s \boldsymbol{r}(1, t)$, so that $v_{k}(1, t)=\boldsymbol{r}(1, t) \cdot \boldsymbol{i}_{k}$. This means that the reduced motion is governed by ordinary differential equations provided that

$$
\mathbf{v}(1, t) \times \hat{\mathbf{n}}\left(\mathbf{0}, \mathbf{v}(1, t), \mathbf{0}, \partial_{t} \mathbf{v}(1, t)\right)=\mathbf{0},
$$

which is required by (6.16) and (2.20), and provided that compatible initial conditions hold. Condition (6.19) says how the shears have to be related to the elongation in such a motion. In particular, if $\hat{n}_{1}, \hat{n}_{2}$ vanish when $v_{1}, v_{2}, \dot{v}_{1}, \dot{v}_{2}$ vanish, then the rod admits a purely longitudinal reduced motion.

Now suppose merely that $d_{3}=i_{3}$, in which case

$$
\boldsymbol{u}(s, t)=u_{3}(s, t) \boldsymbol{i}_{3}
$$

and $\mathbf{D}$ is a rotation about $\boldsymbol{i}_{3}$. Such motions include stretching, shearing, and twisting. We now assume that the material is isotropic (cf. [2]) so that the right-hand sides of (6.18) have the form

$$
\begin{gathered}
\hat{\mathbf{m}}\left(\mathbf{D}(s, t) \cdot \mathbf{u}(1, t), \mathbf{D}(s, t) \cdot \mathbf{v}(1, t), \mathbf{D}(s, t) \cdot \mathbf{u}_{t}(1, t), \mathbf{D}(s, t) \cdot \mathbf{v}_{t}(1, t)\right) \\
=\hat{\mathbf{m}}\left(\mathbf{u}(1, t), \mathbf{D}(s, t) \cdot \mathbf{v}(1, t), \mathbf{u}_{t}(1, t), \mathbf{D}(s, t) \cdot \mathbf{v}_{t}(1, t)\right), \ldots
\end{gathered}
$$

Here we have exploited the form of $\mathbf{D}$ and $\boldsymbol{u}$. This equation admits the solution

$$
\begin{aligned}
& \left(\mathbf{u}(s, t), \mathbf{v}(s, t), \mathbf{u}_{t}(s, t), \mathbf{v}_{t}(s, t)\right) \\
& \quad=\left(u_{3}(1, t) \mathbf{i}_{3}, \mathbf{D}(s, t) \cdot \mathbf{v}(1, t), \partial_{t} u_{3}(1, t) \mathbf{i}_{3}, \mathbf{D}(s, t) \cdot \mathbf{v}_{t}(1, t)\right),
\end{aligned}
$$


so that

$$
\boldsymbol{r}_{s}(s, t)=\boldsymbol{r}_{s}(1, t)
$$

As in (6.2) we immediately integrate $(6.21 \mathrm{~b})$ to get the desideratum $\boldsymbol{r}_{s}(1, t)=\boldsymbol{r}(1, t)$. Combining (6.20) with $(2.3 \mathrm{a})$, we obtain

$$
\partial_{s} \boldsymbol{d}_{1}(s, t)=u_{3}(1, t) \boldsymbol{d}_{2}(s, t), \quad \partial_{s} \boldsymbol{d}_{2}(s, t)=-u_{3}(1, t) \boldsymbol{d}_{1}(s, t) .
$$

The solution of $(6.22)$ that satisfies $(2.9 \mathrm{~b})$ is

$$
\begin{aligned}
& \boldsymbol{d}_{1}(s, t)=\cos \left(s u_{3}(1, t)\right) \boldsymbol{i}_{1}+\sin \left(s u_{3}(1, t)\right) \boldsymbol{i}_{2}, \\
& \boldsymbol{d}_{2}(s, t)=-\sin \left(s u_{3}(1, t)\right) \boldsymbol{i}_{1}+\cos \left(s u_{3}(1, t)\right) \boldsymbol{i}_{2},
\end{aligned}
$$

These equations for $s=1$ give $u_{3}(1, t)$ (nonuniquely) in terms of the components of the $\boldsymbol{d}_{k}(1, t)$. Under our assumptions, the reduced motion is governed by ordinary differential equations provided that the analog of (6.19) and compatible initial conditions hold.

These ordinary differential equations for the rigid body attached to a viscoelastic rod play a less important role than do the ordinary differential equations for the rigid body attached to an elastic rod, because the motions in the former case are much more restricted than those in the latter case. On the other hand, as we now show, we can solve the reduced equations for a viscoelastic rod for any initial conditions.

7. Solvability of the reduced problem for viscoelastic rods. Now we give conditions ensuring that the system (5.1), (5.3), (5.5), (5.6), and (5.7) for the unknowns

$$
\left\{\mathbf{u}(s, \cdot), \mathbf{v}(s, \cdot), \boldsymbol{r}(s, \cdot), \boldsymbol{d}_{k}(s, \cdot): s \in[0,1]\right\}, \quad \boldsymbol{m}(1, \cdot), \quad \boldsymbol{n}(1, \cdot)
$$

admits a globally defined solution for all initial data. (We would relegate this existence theory to an appendix, were it not for the fact that the main ideas are not only elementary, but also illuminate the underlying mechanics.) The technique we use is identical to that for ordinary differential equations: We may use the Contraction Mapping Principle to get a local existence theory, and then we show that the solution can be continued for all time by showing that each of the unknown functions is pointwise bounded for any finite time (see [11, Chap. 6]). In the present setting we seek classical solutions, in which the variables have as many continuous derivatives as appear in the equations. We thus formulate the problem by integrating our differential equations with respect to time and seeking solutions that for fixed $t$ lie in the classical spaces of continuous or continuously differentiable functions of $s$ on $[0,1]$. Since the use of the Contraction Mapping Principle is standard, we limit our attention to obtaining the bounds on (7.1) needed to ensure the global continuation of solutions of (5.1), (5.3), (5.5)-(5.7).

Bounds. We set $\varepsilon=0$ in (2.13) and (2.14), take the dot product of (2.13) with $\boldsymbol{r}_{t}$, take the dot product of (2.14) with $\boldsymbol{w}$, add the resulting equations, integrate the sum with respect to $s$ over $(0,1)$, and then use the boundary conditions $(2.9),(2.15),(2.16)$ 
and the kinematic relations $(2.4),(2.5)$ to obtain

$$
\begin{aligned}
& 0= \int_{0}^{1}\left[\boldsymbol{r}_{t} \cdot \boldsymbol{n}_{s}+\boldsymbol{w} \cdot\left(\boldsymbol{m}_{s}+\boldsymbol{r}_{s} \times \boldsymbol{n}\right)\right] d s \\
&=- \boldsymbol{r}_{t}(1, t) \cdot \boldsymbol{n}(1, t)-\boldsymbol{w}(1, t) \cdot \boldsymbol{m}(1, t) \\
& \quad+\int_{0}^{1}\left[n_{k} \boldsymbol{d}_{k} \cdot\left(v_{l} \boldsymbol{d}_{l}\right)_{t}+\boldsymbol{m} \cdot \boldsymbol{w}_{s}-\boldsymbol{w} \cdot\left(\boldsymbol{r}_{s} \times \boldsymbol{n}\right)\right] d s \\
&=\mu \boldsymbol{r}_{t}(1, t) \cdot\left[\boldsymbol{r}_{t t}(1, t)+\boldsymbol{c}_{t t}(t)\right]+\mu \boldsymbol{w}(1, t) \cdot\left\{\boldsymbol{c}(t) \times\left[\boldsymbol{r}_{t t}(1, t)+\boldsymbol{c}_{t t}(t)\right]\right\} \\
& \quad+\boldsymbol{w}(1, t) \cdot[\boldsymbol{J}(t) \cdot \boldsymbol{w}(1, t)]_{t}+\int_{0}^{1}\left[n_{k} \partial_{t} v_{k}+m_{k} \partial_{t} u_{k}\right] d s \\
&=\frac{1}{2} \frac{d}{d t}\left\{\mu\left|\boldsymbol{r}_{t}(1, t)+\boldsymbol{c}_{t}(t)\right|^{2}+\boldsymbol{w}(1, t) \cdot \boldsymbol{J}(t) \cdot \boldsymbol{w}(1, t)\right\} \\
& \quad+\frac{d}{d t} \int_{0}^{1} W(\mathbf{u}, \mathbf{v}, s) d s \\
& \quad+\int_{0}^{1}\left\{\left[\hat{\mathbf{m}}\left(\mathbf{u}, \mathbf{v}, \mathbf{u}_{t}, \mathbf{v}_{t}, s\right)-\hat{\mathbf{m}}(\mathbf{u}, \mathbf{v}, \mathbf{0}, \mathbf{0}, s)\right] \cdot \mathbf{u}_{t}\right. \\
&\left.\quad+\left[\hat{\mathbf{n}}\left(\mathbf{u}, \mathbf{v}, \mathbf{u}_{t}, \mathbf{v}_{t}, s\right)-\hat{\mathbf{n}}(\mathbf{u}, \mathbf{v}, \mathbf{0}, \mathbf{0}, s)\right] \cdot \mathbf{v}_{t}\right\} d s
\end{aligned}
$$

Integrating (7.2) from 0 to $t$ we get the energy equation:

$$
\begin{aligned}
& \int_{0}^{t} \int_{0}^{1}\left\{\left[\hat{\mathbf{m}}\left(\mathbf{u}, \mathbf{v}, \mathbf{u}_{t}, \mathbf{v}_{t}, s\right)-\hat{\mathbf{m}}(\mathbf{u}, \mathbf{v}, \mathbf{0}, \mathbf{0}, s)\right] \cdot \mathbf{u}_{t}\right. \\
& \left.\quad+\left[\hat{\mathbf{n}}\left(\mathbf{u}, \mathbf{v}, \mathbf{u}_{t}, \mathbf{v}_{t}, s\right)-\hat{\mathbf{n}}(\mathbf{u}, \mathbf{v}, \mathbf{0}, \mathbf{0}, s)\right] \cdot \mathbf{v}_{t}\right\} d s d \tau \\
& \quad+\int_{0}^{1} W(\mathbf{u}(s, t), \mathbf{v}(s, t), s) d s+\frac{1}{2}\left\{\mu\left|\boldsymbol{r}_{t}(1, t)+\boldsymbol{c}_{t}(t)\right|^{2}+\boldsymbol{w}(1, t) \cdot \boldsymbol{J}(t) \cdot \boldsymbol{w}(1, t)\right\} \\
& \quad=\int_{0}^{1} W(\mathbf{u}(s, 0), \mathbf{v}(s, 0), s) d s+\frac{1}{2}\left\{\mu\left|\boldsymbol{r}_{t}(1,0)+\boldsymbol{c}_{t}(0)\right|^{2}+\boldsymbol{w}(1,0) \cdot \boldsymbol{J}(0) \cdot \boldsymbol{w}(1,0)\right\}
\end{aligned}
$$

We assume that the initial data are sufficiently well behaved for the right-hand side of (7.3) to be finite.

We adopt the convention that $K$ denotes a positive constant that can be expressed in terms of the data and that has a meaning that can change in every appearance. An inequality of the form $f(t) \leq K$ tacitly means that there exists such a $K$ for which this inequality holds for all time $t$ for which the solution exists. Since each term on the left-hand side of (7.3) is nonnegative and since $\boldsymbol{J}$ is positive-definite, we conclude that

$$
|\boldsymbol{w}(1, t)| \leq K, \quad\left|\boldsymbol{r}_{t}(1, t)+\boldsymbol{c}_{t}(t)\right| \leq K
$$

from which it follows that

$$
\begin{aligned}
\left|\boldsymbol{c}_{t}(t)\right|=|\boldsymbol{w}(1, t) \times \boldsymbol{c}(t)| & \leq K, \\
\left|\boldsymbol{r}_{t}(1, t)\right| & \leq K, \\
|\boldsymbol{r}(1, t)| & \leq K(1+t), \\
\left|\partial_{t} \boldsymbol{d}_{k}(1, t)\right|=\left|\boldsymbol{w}(1, t) \times \boldsymbol{d}_{k}(1, t)\right| & \leq K .
\end{aligned}
$$


We immediately deduce from (2.34) and (7.3) that

$$
\int_{0}^{t} \int_{0}^{1}\left[\left|\mathbf{u}_{t}\right|+\left|\mathbf{v}_{t}\right|\right] d s d \tau \leq K
$$

Let us assume that our reduced initial-boundary-value problem has a classical solution defined for $t$ in $[0, T)$. We accordingly assume that (7.3) and (7.4) are valid for $t$ in this range. In view of the remarks following (7.1), we can show that the solution can be continued for all time by showing that $\mathbf{u}, \mathbf{v}, \mathbf{u}_{t}, \mathbf{v}_{t}, 1 / \delta^{\sharp}(\mathbf{u}, \mathbf{v}, \cdot), \boldsymbol{m}(1, \cdot), \boldsymbol{n}(1, \cdot)$ cannot blow up pointwise as $t \rightarrow T$.

7.5. Theorem. Let the reduced initial-boundary-value problem (5.1), (5.3), (5.5)-(5.7) have a classical solution defined for $t$ in $[0, T)$. Let $(2.24),(2.26)$, and (2.34) hold. Then $\mathbf{u}, \mathbf{v}, \mathbf{u}_{t}, \mathbf{v}_{t}, 1 / \delta^{\sharp}(\mathbf{u}, \mathbf{v}, \cdot), \boldsymbol{m}(1, \cdot), \boldsymbol{n}(1, \cdot)$ are uniformly bounded for $0 \leq t<T$, so that the classical solution can be continued for all time.

Proof. Relations (2.4b), (2.28), and (7.3) imply that

$$
|\boldsymbol{r}(s, t)| \leq \int_{0}^{1}|\mathbf{v}(s, t)| d s \leq C+\frac{1}{c} \int_{0}^{1} W(\mathbf{u}(s, t), \mathbf{v}(s, t), s) d s \leq K
$$

for all $(s, t) \in[0,1] \times[0, T)$. Thus $\boldsymbol{r}$ is uniformly bounded on $[0,1] \times[0, T)$.

Now we assert that $\boldsymbol{m}$ and $\boldsymbol{n}$ are uniformly bounded on $[0,1] \times[0, T)$. If not, there would be a sequence $\left\{\left(s_{j}, t_{j}\right)\right\}$ in $[0,1] \times[0, T)$ with $t_{j} \rightarrow T$ such that

$$
\left|\boldsymbol{m}\left(s_{j}, t_{j}\right)\right|+\left|\boldsymbol{n}\left(s_{j}, t_{j}\right)\right| \rightarrow \infty .
$$

The integrals (2.42) and the bound (7.6) would then imply that

$$
\left|\boldsymbol{m}\left(s, t_{j}\right)\right|+\left|\boldsymbol{n}\left(s, t_{j}\right)\right| \rightarrow \infty \quad \forall s .
$$

By (2.23) and (2.34), this can only happen if

$$
\left|\mathbf{u}\left(s, t_{j}\right)\right|+\left|\mathbf{v}\left(s, t_{j}\right)\right|+\left|\mathbf{u}_{t}\left(s, t_{j}\right)\right|+\left|\mathbf{v}_{t}\left(s, t_{j}\right)\right|+1 / \delta^{\sharp}\left(\mathbf{u}\left(s, t_{j}\right), \mathbf{v}\left(s, t_{j}\right), s\right) \rightarrow \infty \quad \forall s .
$$

But (2.24), (2.26), (2.34), (7.3) say that this is impossible. Therefore there is a constant $K$ such that

$$
|\boldsymbol{m}(s, t)|+|\boldsymbol{n}(s, t)| \leq K \quad \forall(s, t) \in[0,1] \times[0, T) .
$$

Finally we assert that $\mathbf{u}, \mathbf{v}, \mathbf{u}_{t}, \mathbf{v}_{t}, 1 / \delta^{\sharp}(\mathbf{u}, \mathbf{v}, \cdot)$ are uniformly bounded on $[0,1] \times[0, T)$. If not, there would be a sequence $\left\{\left(s_{j}, t_{j}\right)\right\}$ in $[0,1] \times[0, T)$ with $t_{j} \rightarrow T$ such that

$$
\left|\mathbf{u}\left(s_{j}, t_{j}\right)\right|+\left|\mathbf{v}\left(s_{j}, t_{j}\right)\right|+1 / \delta^{\sharp}\left(\mathbf{u}\left(s_{j}, t_{j}\right), \mathbf{v}\left(s_{j}, t_{j}\right), s_{j}\right) \rightarrow \infty,
$$

which would imply the impossible (7.7).

7.12. TheOREm. Let the hypothesis (2.26) of Theorem 7.5 be replaced with (2.41) and let the initial data have values satisfying (2.7) uniformly. Then the conclusion of Theorem 7.5 is still valid.

Proof. We need only verify that $\delta \equiv \delta^{\sharp}(\mathbf{u}(\cdot, \cdot), \mathbf{v}(\cdot, \cdot), \cdot)$ is bounded away from 0 , since the rest of the proof is exactly the same as that of Theorem 7.5. Without loss of generality, we take $\eta$ smaller than the minimum of the initial values of $\delta$. Suppose that there is an $\left(s_{*}, t_{*}\right)$ such that $\delta\left(s_{*}, t_{*}\right)$ is less than $\eta$. (If there were no such $\left(s_{*}, t_{*}\right)$, then 
there would be nothing to prove.) Since $\delta$ is assumed to be continuous, there is a largest value of $t$, denoted $\tau$, less than $t_{*}$ such that $\delta\left(s_{*}, \tau\right)=\eta$. From (2.15), (2.41), and (2.42a) we obtain

$$
\begin{aligned}
-\Omega_{\delta}\left(\delta\left(s_{*}, t\right), s_{*}\right) \delta_{t}\left(s_{*}, t\right)+C & \geq n_{3}\left(s_{*}, t\right) \\
& =\boldsymbol{n}(1, t) \cdot \boldsymbol{d}_{3}\left(s_{*}, t\right) \\
& =-\mu\left[\boldsymbol{r}_{t t}(1, t)+\boldsymbol{c}_{t t}(t)\right] \cdot \boldsymbol{d}_{3}\left(s_{*}, t\right)
\end{aligned}
$$

for $\tau \leq t \leq t_{*}$. We integrate (7.13) with respect to $t$ over $\left[\tau, t_{*}\right]$ and use (7.4) to obtain

$$
\begin{aligned}
\left.\Omega\left(\delta\left(s_{*}, t\right), s_{*}\right)\right|_{\tau} ^{t_{*}} \leq & \mu \int_{\tau}^{t_{*}}\left[\boldsymbol{r}_{t t}(1, t)+\boldsymbol{c}_{t t}(t)\right] \cdot \boldsymbol{d}_{3}\left(s_{*}, t\right) d t \\
= & \left.\mu\left[\boldsymbol{r}_{t}(1, t)+\boldsymbol{c}_{t}(t)\right] \cdot \boldsymbol{d}_{3}\left(s_{*}, t\right)\right|_{\tau} ^{t_{*}} \\
& \quad-\mu \int_{\tau}^{t_{*}}\left[\boldsymbol{r}_{t}(1, t)+\boldsymbol{c}_{t}(t)\right] \cdot\left[\boldsymbol{w}\left(s_{*}, t\right) \times \boldsymbol{d}_{3}\left(s_{*}, t\right)\right] d t+C\left(t_{*}-\tau\right) \\
\leq & C\left(t_{*}-\tau\right)+2 \mu K+\mu K \int_{\tau}^{t_{*}}\left|w\left(s_{*}, t\right)\right| d t
\end{aligned}
$$

Since (2.5) implies that

$$
\boldsymbol{w}\left(s_{*}, t\right)=\int_{0}^{s_{*}} \boldsymbol{w}_{s}(s, t) d s=\int_{0}^{s_{*}} \partial_{t} u_{k}(s, t) \boldsymbol{d}_{k}(s, t) d s
$$

we use $(7.4 \mathrm{f})$ to obtain

$$
\int_{\tau}^{t_{*}}\left|\boldsymbol{w}\left(s_{*}, t\right)\right| d t \leq \int_{\tau}^{t_{*}} \int_{0}^{1}\left|\mathbf{u}_{t}\right| d s d t \leq K
$$

The substitution of (7.16) into (7.14) shows that the left-hand side of (7.14) is dominated by a number depending on $t_{*}$. The properties of $\Omega$ thus ensure that $\delta$ has a positive lower bound depending on $t_{*}$.

We could easily generalize (2.41) by adding $C \Omega$ to the right-hand side of the inequality. In this case we would obtain the proof of Theorem 7.12 by using the Gronwall Inequality. Condition 2.41 unduly restricts the dependence of $\hat{n}_{3}$ on $\dot{\delta}$ for large positive $\delta$ to be sublinear. This flaw can be corrected by replacing (2.41) with a more subtle system of constitutive hypotheses at the expense of complicating the analysis. See $[3,4]$ for details.

8. Comments. Let $\eta$ be a small positive parameter. We could consider a family of viscoelastic rods with constitutive functions $\hat{\mathbf{m}}$ and $\hat{\mathbf{n}}$ of the form

$$
\hat{\mathbf{m}}(\mathbf{u}, \mathbf{v}, \dot{\mathbf{u}}, \dot{\mathbf{v}}, s)=\frac{\partial W}{\partial \mathbf{u}}(\mathbf{u}, \mathbf{v}, s)+\mathbf{m}^{b}(\mathbf{u}, \mathbf{v}, \eta \dot{\mathbf{u}}, \eta \dot{\mathbf{v}}, s), \ldots
$$

(This is the simplest of many possible ways of introducing a small viscosity parameter $\eta$.) Under the assumption that (2.33) and (2.34) hold, the system (2.36) when (8.1) holds is equivalent to

$$
\eta \dot{\mathbf{u}}=\dot{\mathbf{u}}^{b}\left(\mathbf{u}, \mathbf{v}, \mathbf{m}-\frac{\partial W}{\partial \mathbf{u}}(\mathbf{u}, \mathbf{v}, s), \mathbf{n}-\frac{\partial W}{\partial \mathbf{v}}(\mathbf{u}, \mathbf{v}, s), s\right), \ldots
$$


Therefore, in place of (5.3)-(5.5) we have

$$
\begin{aligned}
\eta \boldsymbol{r}_{t} & =\int_{0}^{s}\left[\dot{v}_{k}^{b} \boldsymbol{d}_{k}+\eta \boldsymbol{w} \times v_{k} \boldsymbol{d}_{k}\right] d \xi \\
\eta \boldsymbol{w}(s, t) & =\int_{0}^{s} \dot{u}_{k}^{b} \boldsymbol{d}_{k} d \xi \\
\partial_{t} \boldsymbol{d}_{k} & =\boldsymbol{w} \times \boldsymbol{d}_{k} .
\end{aligned}
$$

Note that by setting $\eta=0$, we recover the problem for elastic rods treated in Sec. 4 . The analyses of Secs. 3 and 4 suggest that the asymptotics of (8.3)-(8.5) may lead to initial layers and to internal temporal layers at the times at which jumps occur for the problem with $\eta=0$. Let $\bar{t}$ be a time at which such a jump occurs. We introduce the stretched time $\tau$ and new dependent variables by

$$
\tau \equiv \frac{t-\bar{t}}{\eta}, \quad \tilde{\boldsymbol{r}}(s, \tau, \eta) \equiv \boldsymbol{r}(s, \bar{t}+\eta \tau), \ldots
$$

We substitute (8.6) into (8.3)-(8.5) to get an equivalent system, which is important in the layers. Setting $\eta=0$ in this system we get its leading terms:

$$
\tilde{\boldsymbol{r}}_{\tau}=\int_{0}^{s} \dot{v}_{k}^{\mathrm{b}} \tilde{\boldsymbol{d}}_{k} d \xi, \quad \mathbf{0}=\int_{0}^{s} \dot{u}_{k}^{\mathrm{b}} \tilde{\boldsymbol{d}}_{k} d \xi, \quad \partial_{t} \tilde{\boldsymbol{d}}_{k}=\mathbf{0} .
$$

The study of the asymptotics of (8.3)-(8.5) might illuminate and justify the processes described in Sec. 3 (in a way vaguely analogous to that of [13]). Nevertheless, we do not pursue this study here because it seems to be complicated enough to require a separate treatment, and because some of the significance of this study is predicated on the presumption that taking the asymptotic limit $\eta \rightarrow 0$ after the asymptotic limit $\varepsilon \rightarrow 0$ is physically valid.

Indeed, a proper asymptotics should treat the behavior of solutions for all $\varepsilon, \eta \geq 0$ with $\varepsilon, \eta$ small. That such a study might justify the results of Secs. 3 and 4 is suggested by work treating partial differential equations as infinite-dimensional dynamical systems (cf. Ilyashenko [8] and Temam [15], e.g.). For certain partial differential equations with suitable damping, such as the Navier-Stokes equations, this theory makes it possible to estimate the dimension of the attractor; this dimension corresponds to the number of ordinary differential equations in time (generated, e.g., by the Faedo-Galerkin method) necessary to give an effective description of the behavior of solutions. For a special version of the Navier-Stokes equations, Ilyashenko shows that this dimension is $O\left(\eta^{-4}\right)$, which is very large when the viscosity is small. Here $\eta$ is the viscosity, which is just what it is in (8.1).

Now we have shown that by letting the inertia parameter $\varepsilon$ go to zero, we can formally reduce our partial differential equations either to ordinary differential equations or at least to much simpler dynamical systems. Thus the inertia parameter works in oppoosition to the viscosity. Suppose for fixed $\varepsilon$ that the dimension of the attractor is $O\left(\eta^{-\alpha}\right)$ where $\alpha$ is positive and further that when $\varepsilon$ varies, the dimension of the attractor is $O\left((\eta / \varepsilon)^{-\alpha}\right) ; \eta / \varepsilon$ is a specific viscosity. If $\varepsilon=o(\eta)$, then this dimension is the small number $O\left((o(\eta) / \eta)^{\alpha}\right)$. 
There are obvious generalizations of our methods: We could attach any system of rigid bodies, e.g., a gyroscope, to the end. These bodies need not be rigidly attached to the end. The motion of the rigid body can be constrained, e.g., by making a material point slide in a groove. We can apply any system of forces to the rod and to the rigid bodies. The resulting forced motions might well exhibit quite interesting chaotic motions, especially because of the multiplicity of motions we described for elastic rods. We could employ a theory of rods of any complexity.

The problems treated above are far more complicated than that for purely longitudinal motion treated in [1]. Here, elastic rods, driven by the motion of the rigid body, move through multiple equilibrium states. For longitudinal motions, there is a unique equilibrium state determined by the position of the end mass. Our method for showing that the motion of a rigid body held by an elastic rod is governed by ordinary differential equations is completely different from that of [1]. For related questions, see [14]. The full existence theory for all positive $\varepsilon$ appears in [4].

Research being conducted by Antman shows promise of developing a rigorous asymptotics for viscoelastic rods as the inertia parameter $\varepsilon \rightarrow 0$. This problem is much simpler than that of treating the asymptotics as both $\varepsilon$ and a viscosity parameter $\eta$ go to 0 .

The mathematical problems we treat are analogous to those encountered in the study of the motion of free surfaces for perfect fluids. Here, too, the equations of evolution are the boundary conditions.

Acknowledgment. The research of Antman was partially supported by grants from the NSF and AFOSR.

\section{REFERENCES}

[1] S. S. Antman, The paradoxical asymptotic status of massless springs, SIAM J. Appl. Math. 48, 1319-1334 (1988)

[2] S. S. Antman, Nonlinear Problems of Elasticity, Springer-Verlag, New York, 1995

[3] S. S. Antman and T. I. Seidman, Quasilinear hyperbolic-parabolic equations of one-dimensional viscoelasticity, J. Differential Equations 124, 132-185 (1996)

[4] S. S. Antman and T. I. Seidman, Parabolic-hyperbolic systems governing the spatial motion of nonlinearly viscoelastic rods (in preparation)

[5] P. F. Byrd and M. D. Friedman, Handbook of Elliptic Integrals for Engineers and Scientists, 2nd edition, Springer-Verlag, New York, 1971

[6] R. L. Fosdick and R. D. James, The elastica and the problem of pure bending for a non-convex stored energy function, J. Elasticity 11, 165-186 (1981).

[7] R. Frisch-Fay, Flexible Bars, Butterworths, 1962

[8] Yu. S. Ilyashenko, Weakly attracting systems and attractors of Galerkin approximations of NavierStokes equations on the two-dimensional torus, Selecta Math. Sov. 11, 203-239 (1992)

[9] R. D. James, The equilibrium and post-buckling behavior of an elastic curve governed by a nonconvex energy, J. Elasticity 11, 239-269 (1981)

[10] A. E. H. Love, A Treatise on the Mathematical Theory of Elasticity, 4th edition, Dover Publications, New York, 1944

[11] R. H. Martin, Nonlinear Operators and Differential Equations in Banach Spaces, WileyInterscience, New York-London-Sydney, 1976

[12] E. L. Reiss, Column buckling-an elementary example of bifurcation, in Bifurcation Theory and Nonlinear Eigenvalue Problems, J. B. Keller and S. Antman, eds., Benjamin, 1969, pp. 1-16 
[13] E. L. Reiss and B. J. Matkowsky, Nonlinear dynamic buckling of a compressed elastic column, Quart. Appl. Math. 29, 245-260 (1971)

[14] D. Shilkrut, A paradox of the classical approach in the theory of vibrations of geometrically nonlinear elastic systems, Ben Gurion University of the Negev, 1989

[15] R. Temam, Infinite-Dimensional Dynamical Systems in Mechanics and Physics, 2nd edition, Springer-Verlag, New York, 1997

[16] A. H. Zemanian, Distribution Theory and Transform Analysis, McGraw-Hill, New York, 1965 